\title{
Dynamic instability of pile-supported structures in liquefiable soils during earthquakes
}

\author{
S. Adhikari ${ }^{\mathrm{a}, *}$ and S. Bhattacharya ${ }^{\mathrm{b}}$ \\ ${ }^{\text {a }}$ University of Wales Swansea, Swansea, UK \\ ${ }^{\mathrm{b}}$ University of Bristol, Bristol, UK
}

Received 12 April 2007

Revised 18 September 2007

\begin{abstract}
Piles are long slender columns installed deep into the ground to support heavy structures such as oil platforms, bridges, and tall buildings where the ground is not strong enough to support the structure on its own. In seismic prone zones, in the areas of soft soils (loose to medium dense soil which liquefies like a quick sand) piles are routinely used to support structures (buildings/ bridges). The pile and the building vibrate, and often collapse, in liquefiable soils during major earthquakes. In this paper an experimental and analytical approach is taken to characterize this vibration. The emphasis has been given to the dynamic instability of piled foundations in liquefied soil. The first natural frequency of a piled-structure vibrating in liquefiable soil is obtained from centrifuge tests. The experimental system is modelled using a fixed-free Euler-Bernoulli beam resting against an elastic support with axial load and tip mass with rotary inertia. Natural frequencies obtained from the analytical method are compared with experimental results. It was observed that the effective natural frequency of the system can reduce significantly during an earthquake.
\end{abstract}

\section{Introduction}

During strong earthquakes under the action of shear loading, loose to medium dense saturated sands lose strength and liquefy - the phenomenon is called 'liquefaction' and is quite similar to quick sand. In mildly sloping ground, the soil usually flows following the liquefaction. Collapse and/or severe damage of pile-supported structures is still observed in loose to medium dense sands (liquefiable soils) after most major earthquakes such as 1995 Kobe earthquake (Japan), 1999 Koceli earthquake (Turkey), 2001 Bhuj earthquake (India) and the 2005 Sumatra earthquake. The failures not only occurred in sloping grounds but were also observed in level grounds. The failures were often accompanied by settlement and tilting of the superstructure, rendering it either useless or very expensive to rehabilitate after the earthquake, see Fig. 1. Following the 1995 Kobe earthquake, investigation has been carried out to find the failure pattern of the piles, Yoshida and Hamada [41], BTL [32]. Piles were excavated or extracted from the subsoil, borehole cameras were used to take photographs, and pile integrity tests were carried out. These studies hinted the location of the cracks and damage patterns for the piles. Of particular interest is the formation of plastic hinges in the piles. This indicates that the stresses in the pile during and after liquefaction exceeded the yield stress of the material of the pile despite large factors of safety employed in the design. As a result, design of pile foundation in seismically liquefiable areas still remains a constant source of attention to the earthquake geotechnical engineering community.

\footnotetext{
${ }^{*}$ Corresponding author. Chair of Aerospace Engineering, School of Engineering, University of Wales Swansea, Singleton Park, Swansea SA2 8PP, UK. AIAA Senior Member. E-mail: S.Adhikari@swansea.ac.uk.
} 


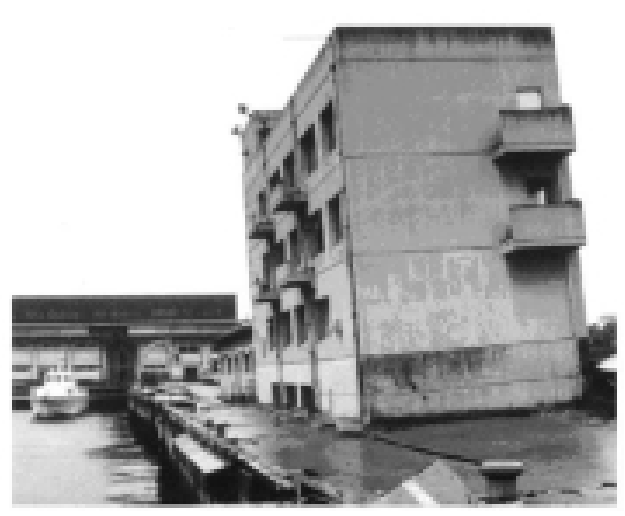

(a) Failure pattern of a pile-supported building, photo courtesy K. Tokimatsu

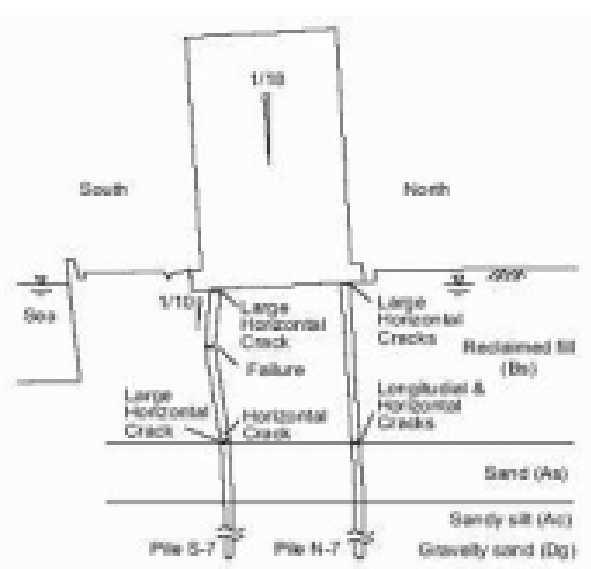

(b) Damage pattern of the piles supporting the building

Fig. 1. The collapse of a building supported on 38 piles in the Higashinada-ku area of Kobe City.

In a recent investigation [26,35] the importance of partial to full loss of lateral support over a portion of the pile length owing to soil liquefaction has been highlighted. It has been concluded that the degradation of the soil strength due to liquefaction has a significant influence on the buckling instability type failure. The study is based on extension of Mindlin solution for a point load acting inside semi-infinite elastic space. This has been also experimentally investigated in references $[6,23]$. However, the dynamics of the pile instability has not been considered in the above study and is the focus of this investigation.

Figure 1(a) shows the collapse of a building supported on 38 piles. The building was located $6 \mathrm{~m}$ from the quay wall on a reclaimed land in Higashinada-ku area of Kobe City. After the 1995 Kobe earthquake, the quay wall was displaced by $2 \mathrm{~m}$ towards the sea and the building tilted by about 3 degrees. Following the earthquake, investigation was carried out to find the damage pattern, see Fig. 1(b). The failure pattern suggests that the building supported on the piles rotated during the earthquake. Therefore the rotary inertia of the building should be accounted for in the analysis. This type of failure pattern could also be replicated in carefully designed small scale model tests carried out by Bhattacharya et al. [6], Knappett and Madabhushi [23] while studying the buckling instability of piled foundations in liquefiable soils. Figure 2 shows the failure pattern of a single pile and a pile group observed in small scale geotechnical centrifuge tests. The key principles of a geotechnical centrifuge are explained in subsection 3.1.

Motivated by the real-life failures of pile-supported structures, together with the experimental evidences, an unified approach comprising of bending, buckling and dynamics has been proposed in this paper. An Euler-Bernoulli beam model resting against an elastic support with axial force and tip mass with rotary inertia is considered. The elastic support is aimed at modeling the surrounding soil while the tip mass together with its rotary inertia is aimed at modeling the superstructure. Only free vibration analysis is considered in this work. In Section 2 a brief review on the cause of failure of piled foundation during earthquakes is given. In Section 3 the experimental adopted in this study is explained and selected results are presented. A beam model with tip mass and rotary inertia is analyzed in Section 4. Exact analytical expressions to obtain the natural frequencies of the combined system are derived in terms of non-dimensional parameters describing the system. Numerical Results obtain using the proposed approach are presented in Section 5 .

\section{Current understanding of the cause of failure of piled foundation during earthquakes}

Figure 3 shows the different stages of loading on a pile-supported foundation during an earthquake. Stage I in the figure describes the load sharing between the shaft resistance (shear generated along the surface of the pile) and 


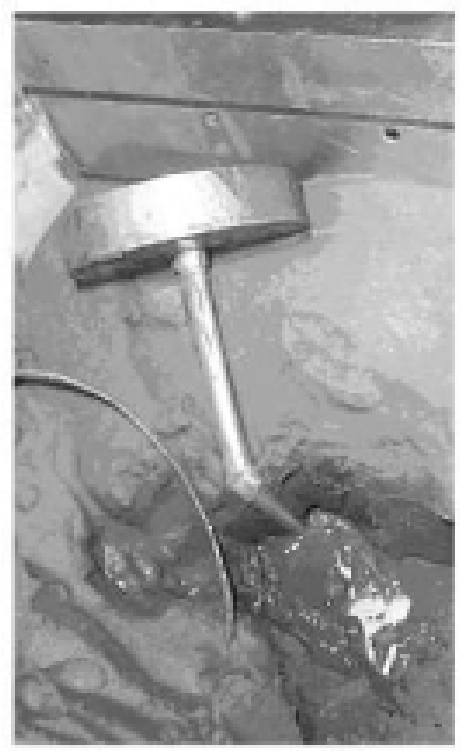

(a) Buckling of a single pile in a centrifuge test, Bhattacharya [2]

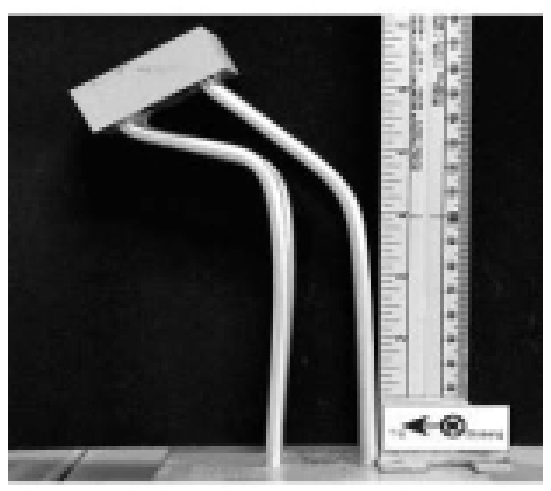

(b) Buckling of apile group in acentrifuge test, Knappett and Madabhushi [23]

Fig. 2. Buckling pattern of single pile and a pile group in a centrifuge test.

end-bearing of the pile (bearing of the tip of the pile) in normal condition i.e. prior to an earthquake. The vertical load of the building which can be considered purely static $\left(P_{\text {static }}\right)$ is carried by the shear (friction along the length of the pile) and end-bearing in the pile. However, during earthquakes, soil layers overlying the bedrock are subjected to seismic excitation consisting of numerous incident waves, namely shear $(\mathrm{S})$ waves, dilatational or pressure $(\mathrm{P})$ waves, and surface (Rayleigh and Love) waves which result in ground motion. The ground motion at a site will depend on the stiffness characteristics of the layers of soil overlying the bedrock. This motion will also affect a piled structure. As the seismic waves arrive in the soil surrounding the pile, the soil layers will tend to deform. This seismically deforming soil will try to move the piles and the embedded pile-cap with it. Subsequently, depending upon the rigidity of the superstructure and the pile-cap, the superstructure may also move with the foundation. The pile may thus experience two distinct phases of initial soil-structure interaction.

1. Before the superstructure starts oscillating, the piles may be forced to follow the soil motion, depending on the flexural rigidity (EI) of the pile. Here the soil and pile may take part in kinematic interplay and the motion of the pile may differ substantially from the free field motion. This may induce bending moments in the pile.

2. As the superstructure starts to oscillate, inertial forces are generated. These inertia forces are transferred as lateral forces and overturning moments to the pile via the pile-cap. The pile-cap transfers the moments as varying axial loads and bending moments in the piles. Thus the piles may experience additional axial and lateral loads, which cause additional bending moments in the pile.

These two effects occur with only a small time lag and have been studied in some detail by Tokimatsu and Asaka [37]. If the section of the pile is inadequate, bending failure may occur in the pile. The behaviour of the pile at this stage may be approximately described as a beam on an elastic foundation, where the soil provides sufficient lateral restraint. The available confining pressure around the pile is not expected to decrease substantially in these initial phases. The response to changes in axial load in the pile would not be severe either, as shaft resistance continues to act. This is shown in Fig. 3 (Stage II).

In loose saturated sandy soil, as the shaking continues, pore pressure will build up and the soil will start to liquefy. With the onset of liquefaction, an end-bearing pile passing through liquefiable soil will experience distinct changes in its stress state. 


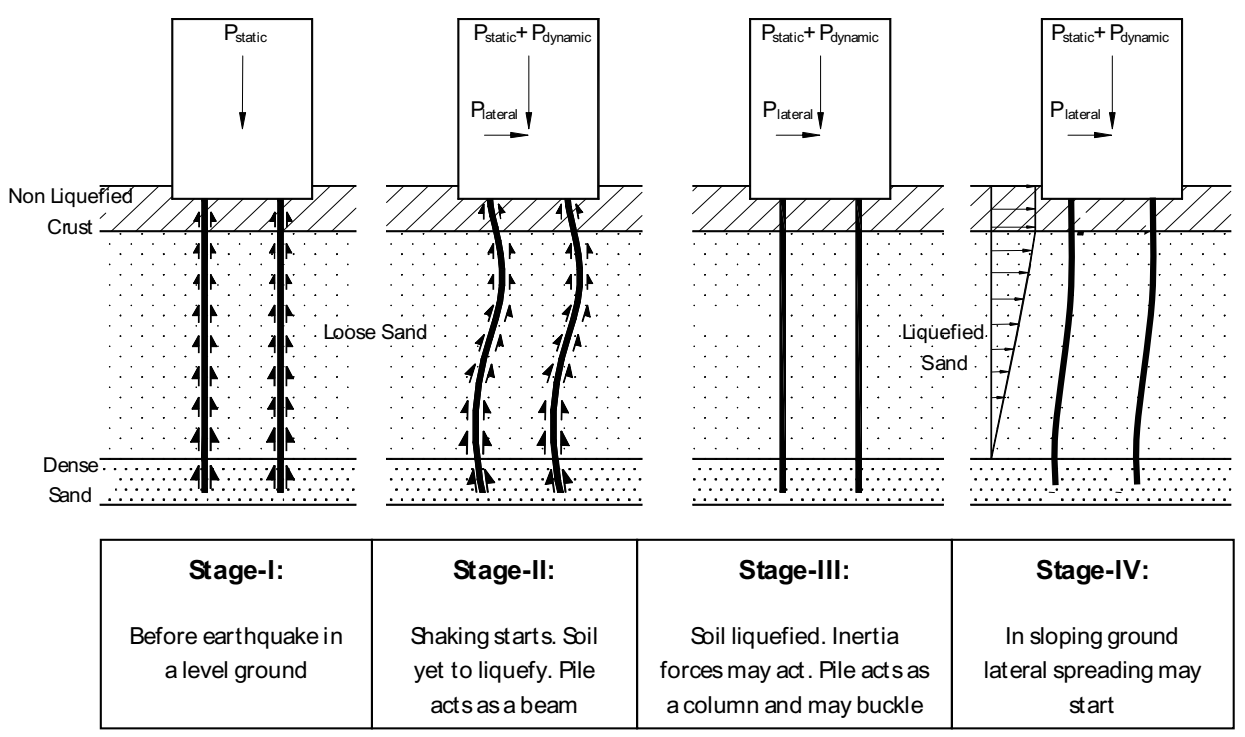

Fig. 3. Different stages of the loading on a pile in liquefiable soil.

- The pile will start to lose its shaft resistance in the liquefied layer and shed axial loads downwards to mobilise additional base resistance. If the base capacity is exceeded, settlement failure will occur.

- The liquefied soil will begin to lose its stiffness so that the pile acts as an unsupported column as shown in Fig. 3 (Stage III). Piles that have a high slenderness ratio will then be prone to axial instability, and buckling failure will occur in the pile, enhanced by the actions of lateral disturbing forces and also by the deterioration of bending stiffness due to the onset of plastic yielding, see Bhattacharya et al. [5,7], Bhattacharya et al. [6]. This particular mechanism is currently missing in all codes of practice and has been described as a fundamental omission in seismic pile design, Bhattacharya and Bolton [4].

In sloping ground, even if the pile survives the above load conditions, it may experience additional drag load due to the lateral spreading of soil. Under these conditions, the pile may behave as a beam-column (column with lateral loads); see Fig. 3 (Stage IV). This bending mechanism is currently considered most critical for pile design, see for example Japanese Road Association code (2002), Eurocode 8.

After some initial time period, as the soil starts liquefying (Stage III in Fig. 3), the motion of the pile will be a coupled motion. This coupling will consist of: (a) transverse static bending predominantly due to the lateral loads, (b) dynamic buckling arising due to the dynamic vertical load of the superstructure, and (c) motion due to dynamic amplification caused by the frequency dependent force arising due to the shaking of the bedrock and its surroundings. In the initial phase (Stage II in Fig. 3), when the soil has not fully liquefied, the transverse static bending is expected to govern the internal stresses within the pile. As the liquefaction progresses, the coupled buckling and resonance would govern the internal stresses and may eventually lead to dynamic failure. The key physical aspect that the authors aim to emphasize is that the motion of the pile (and consequently the internal stresses leading to the failure) is a coupled phenomenon. This coupling is, in general, nonlinear and it is not straightforward to exactly distinguish the contributions of the different mechanisms towards an observed failure. It is however certainly possible that one mechanism may dominate over the others at a certain point of time during the period of earthquake motion and till the dissipation of excess pore water pressure. A coupled dynamical analysis combining (a) transverse static bending, (b) buckling instability and (c) dynamic amplification (near resonance) must be carried out for a comprehensive understanding of the failure mechanism of piles during an earthquake. The purpose of this paper is therefore to understand the vibrational characteristics of the piled foundation at full liquefaction i.e. the time instant shown by Stage III in Fig. 3. This has design implications as it is necessary to predict the lateral and vertical dynamic loads in the pile at full liquefaction. 
Table 1

Scaling laws in a centrifuge test

\begin{tabular}{lcc}
\hline Parameter & Model/prototype & Dimensions \\
\hline Length & $1 / N$ & $\mathrm{~L}$ \\
Mass & $1 / N^{3}$ & $\mathrm{M}$ \\
Stress & 1 & $\mathrm{ML}^{-1} \mathrm{~T}^{-2}$ \\
Strain & 1 & 1 \\
Force & $1 / N^{2}$ & $\mathrm{MLT}^{-2}$ \\
Seepage velocity & $N$ & $\mathrm{LT}^{-1}$ \\
Time (seepage) & $1 / N^{2}$ & $\mathrm{~T}$ \\
Time (dynamic) & $1 / N$ & $\mathrm{~T}$ \\
Frequency & $N$ & $1 / \mathrm{T}$ \\
Acceleration & $N$ & $\mathrm{LT}^{-2}$ \\
Velocity & 1 & $\mathrm{LT}^{-1}$ \\
EI (bending stiffness) & $1 / N^{4}$ & $\mathrm{ML}^{3} \mathrm{~T}^{-2}$ \\
MP (plastic moment capacity) & $1 / N^{3}$ & $\mathrm{ML}^{2} \mathrm{~T}^{-2}$ \\
\hline
\end{tabular}

Table 2

Information on the pile section

\begin{tabular}{lc}
\hline Outside diameter & $9.3 \mathrm{~mm}$ \\
Inside diameter & $8.5 \mathrm{~mm}$ \\
Material & Dural alloy \\
Stiffness & $7.77 \times 10^{6} \mathrm{~N} \mathrm{~mm}{ }^{2}$ \\
Length & $189 \mathrm{~mm}$ \\
Mass per unit length & $0.3 \mathrm{gm} / \mathrm{mm}$ \\
\hline
\end{tabular}

\section{Experimental analysis of vibration of piled foundations}

\subsection{Centrifuge modelling}

In soil mechanics or geotechnical engineering, model tests using small size models (1:N where $\mathrm{N}$ is the scaling ratio) under 1-g conditions cannot reproduce the prototype behaviour because the stress level due to self-weight is much lower than that in the field scale prototype. The behaviour of soils has been established to be highly non-linear and hence true prototype behaviour can only be observed in a model under stress and strain conditions similar to the prototype. A geotechnical centrifuge enables us to recreate the same stress and strain level within the scaled model by testing a $1: N$ scale model at $N$ times earth's gravity, created by centrifugal force.

In the centrifuge, the linear dimensions are modelled by a factor $1 / \mathrm{N}$ and the stress is modelled by a factor of unity. Scaling laws for many parameters in the model can be obtained by simple dimensional analysis, and are discussed by Schofield $[33,34]$ as summarised in Table 1.

\subsection{Experimental investigation of vibration of a single pile in liquefied soil}

Dynamic centrifuge tests were carried out at Schofield Centre (University of Cambridge) to verify that fully embedded piles, passing through saturated, loose to medium dense sands, and end-bearing on hard layers, buckle under the action of axial load alone if the surrounding soil liquefies in an earthquake, see Bhattacharya [2]. During earthquakes, whether at model or field scale, the axial loads on a pile are accompanied by lateral loads induced by the inertia of the superstructure and the drag of laterally spreading soil. The failure of a pile can arise because of any one of these load effects, or a suitable combination of them. The centrifuge tests were designed in level ground to avoid the effects of lateral spreading. Twelve piles were tested in a series of four centrifuge tests including some which decoupled the effects of inertia and axial load. The model piles were made of dural alloy tube having an outside diameter of $9.3 \mathrm{~mm}$, a thickness of $0.4 \mathrm{~mm}$ and a total length of $160 \mathrm{~mm}$ or $180 \mathrm{~mm}$. Properties of the model pile can be seen in Table 2.

The sand used to build the models was Fraction E silica sand, which is quite angular with D50 grain size of $0.14 \mathrm{~mm}$, maximum and minimum void ratio of 1.014 and 0.613 respectively, and a specific gravity of 2.65 . Axial load (P) was applied to the pile through a block of brass fixed at the pile head (see Fig. 2(a)). With the increase in 


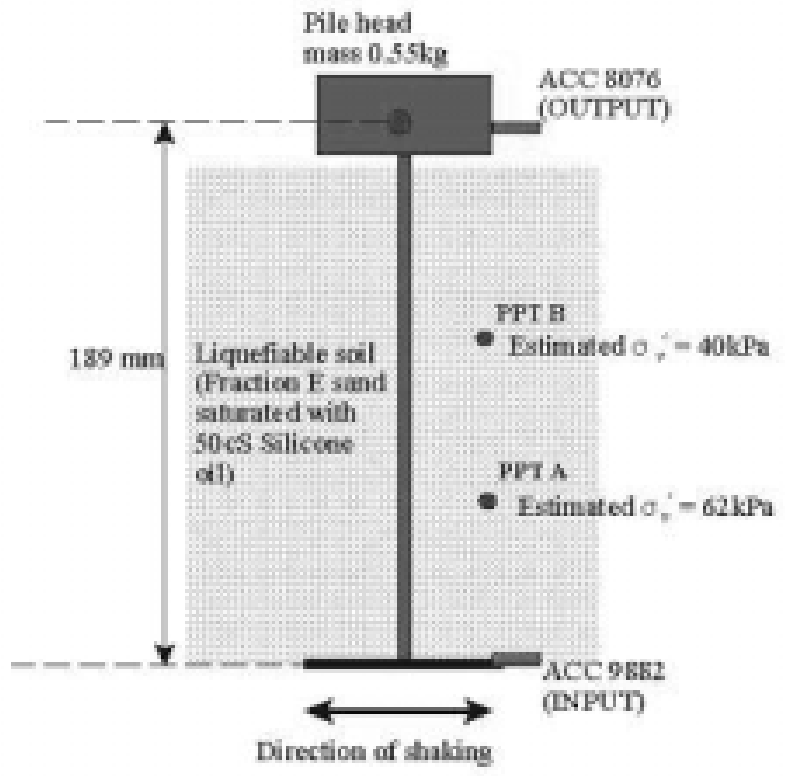

Fig. 4. SDOF oscillator showing the locations of the accelerometers and the PPT's.

centrifugal acceleration, the brass weight imposes increasing axial load in the pile. The packages were centrifuged to $50 \mathrm{~g}$, one-dimensional earthquakes were fired and the soil liquefied. Details of the early tests can be found in Bhattacharya [2] and Bhattacharya et al. [6], while this paper details test results of a single pile which was aimed at understanding the vibration characteristics when soil liquefies.

A cantilever column with a tip mass is the simplest form of a vibrating system. As there is only boundary condition (i.e. the fixed end) to be simulated, the problem can also be studied experimentally without much error. Details of the experimental set-up can be seen in Bhattacharya et al. [6]. Figure 4 shows the schematic of the simple experiment.

The external excitation, i.e. the time-varying force acting on the pile-toe is due to the earthquake, is measured by accelerometer ACC 9882. This input motion is shown in Fig. 5(a). In Fig. 5(b) we have also shown the time-history of the output motion measured at the pile-head.

The FFT of the input signal provided by the actuator is shown in Fig. 6.

As the experiment was carried out at 50-g (fifty times earth's gravity), this input motion represent a prototype earthquake of $1 \mathrm{~Hz}$ frequency (see Table 1 for scaling law). The time history of the loading shows that there were two excitations:

1. Excitation 1 (between 0.25 seconds and 1.25 seconds) when the soil was initially solid and then transformed into a liquefied mass. Study of the pore pressure response in the experiment suggests that the soil liquefied just after 0.3 seconds i.e. after two full cycles of loading. This earthquake corresponds to a prototype earthquake of 50 seconds duration (see scaling law for dynamic time in Table 1).

2. Excitation 2 (between 3.5 seconds and 6 seconds) when the soil was fully liquefied.

\subsection{Data acquisition and the test results}

Accelerometers and Pore Pressure transducers were used to record the responses during the vibration. Data was recorded for 6 seconds at the rate of $4000 \mathrm{~Hz}$ i.e. 24000 data was acquired. The accelerometer (ACC 8076) at the pile head records the responses in the pile head i.e. the transfer of input acceleration to the pile head as soil liquefies due to the stiffness degradation of the pile-soil system, referred herein as 'output'. The pile head mass was not in contact with the liquefied ground and therefore the response is due to combined stiffness of the pile and the soil. This is function of the stiffness and damping of the pile-soil system. Figure 7 shows the traces of the excess pore pressure generated in the soil during the earthquake which provides us information regarding the onset 


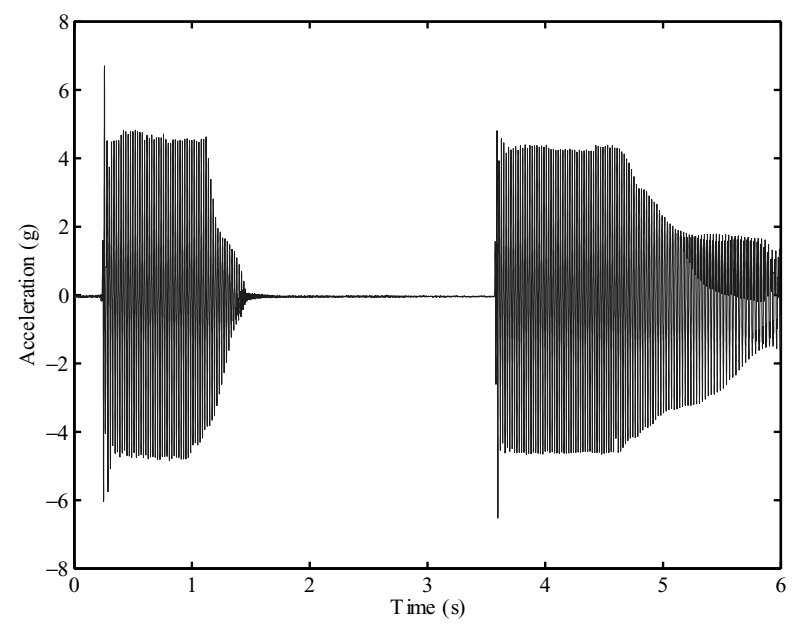

(a) Time history of the input acceleration (ACC 9882) at the pile-toe

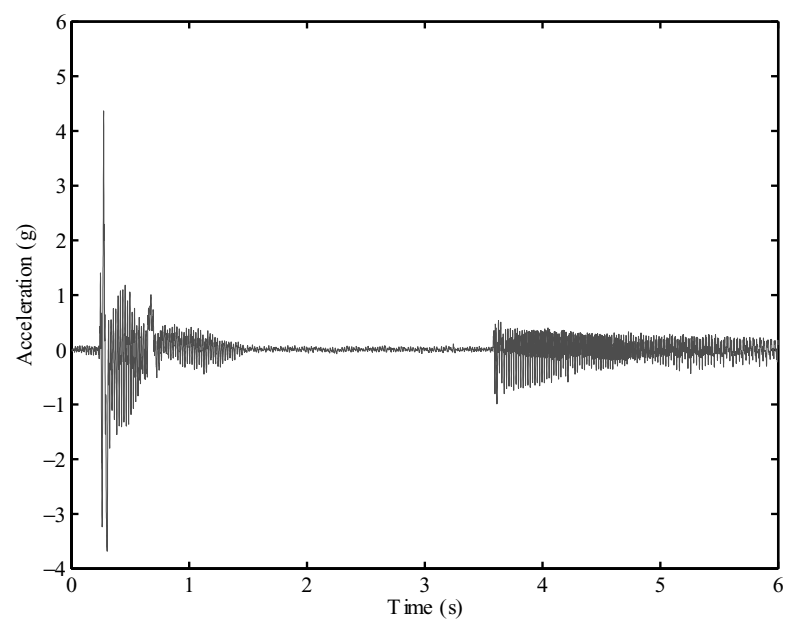

(b) Time history of the output acceleration at the pile-head (the unit of acceleration is $g$ )

Fig. 5. Time histories of the input and output acceleration.

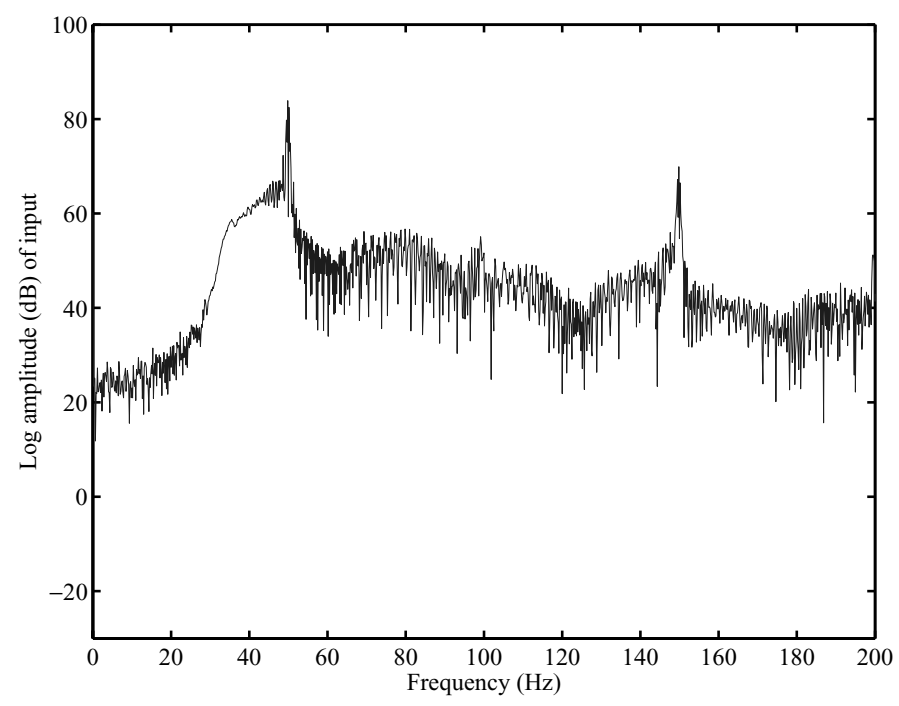

Fig. 6. FFT of the input signal.

of liquefaction. Liquefaction is defined as the point when the pore water pressure reaches the total vertical stress of the soil or effective vertical stress is zero. It may be noted that as the shaking starts the excess pore pressure rises in the soil and reached a plateau at about 0.32 seconds. Figure 7 suggests that in each case, the plateau corresponds well with an estimate of the pre-existing effective vertical stress at the corresponding elevation, suggesting that the vertical effective stress had fallen to zero. In other words, the soil had liquefied. Between 0.32 second and 0.6 seconds in Fig. 7, the pile would have lost all lateral effective stress from the soil i.e. the bracing action of the soil against buckling is almost negligible. Horizontal arrows in the right-hand side of Fig. 7 represent the excess pore pressure at which the vertical effective stress equals to zero for each PPT. It is interesting to note the change in the amplitude of vibration at the pile head during this period, see Fig. 8. The present paper is intended to improve understanding of this vibration problem when the soil liquefies.

From the acceleration record (see Fig. 8) it can be observed that the motion of the pile is initially in phase, i.e. in 


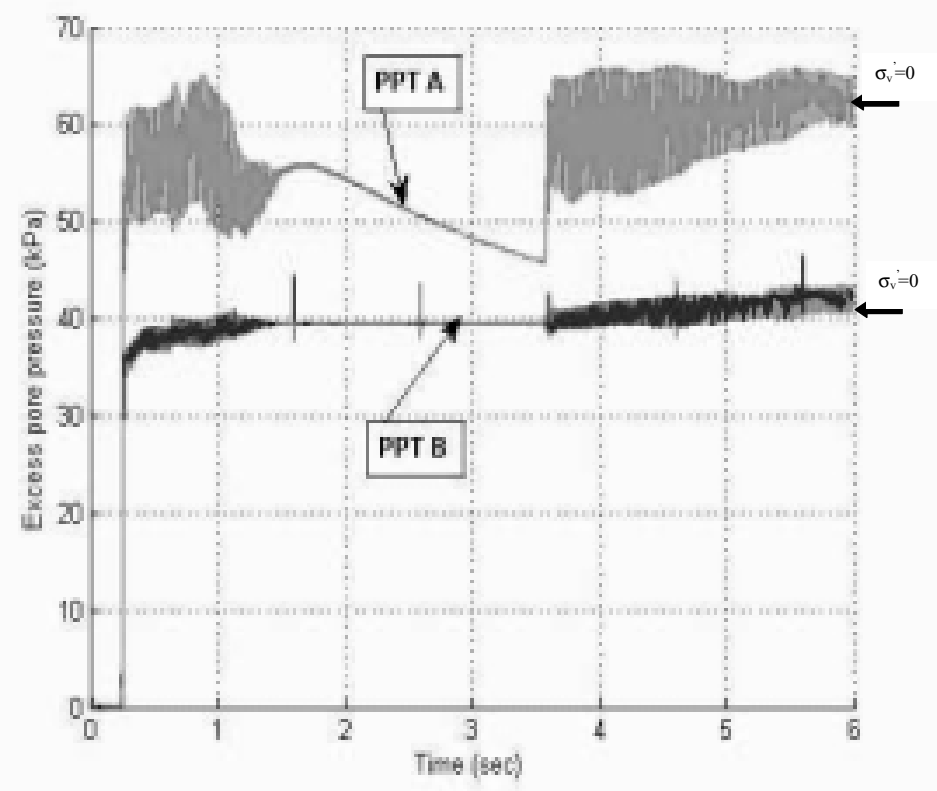

Fig. 7. Excess pore pressure generation and dissipation in the test.

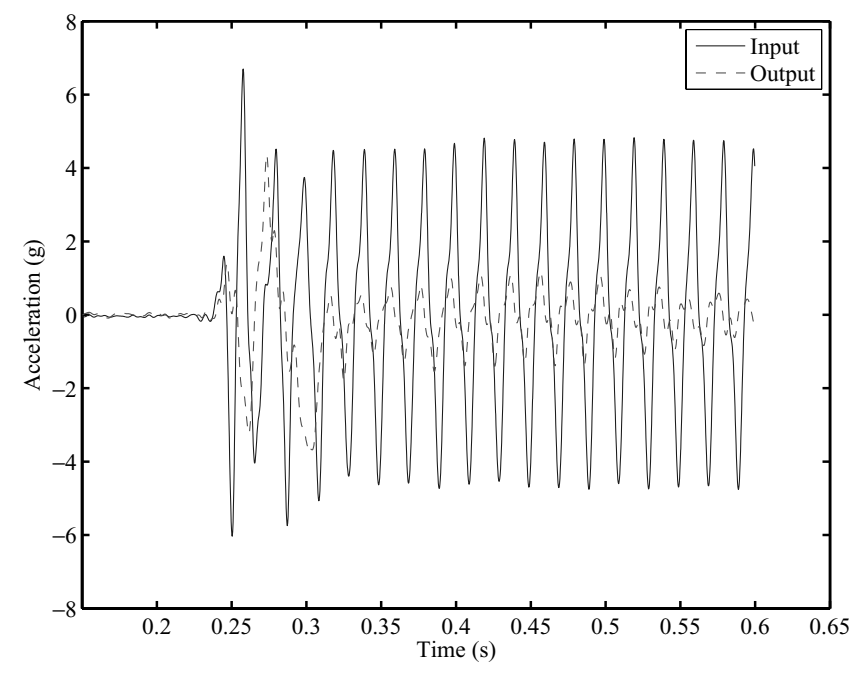

Fig. 8. Input acceleration and the transmitted acceleration to the pile head.

the first half cycle before soil starts to liquefy and during shaking. With the progression of the rise of pore pressure i.e. liquefaction in a top-down fashion, the stiffness of the pile-soil system decreases and the motion of the pile is out of phase. After full liquefaction, i.e. just after the instant of $0.33 \mathrm{sec}$ in the time record, the motion of the pile comes in phase with the input acceleration.

Figure 9(a) shows the frequency response function of the system corresponding to time domain data shown in Fig. 8. This is essentially the ratio of the output and input of the pile plotted in the frequency domain. Coherence corresponding to this frequency response function is shown in Fig. 9(b) The coherence is reasonably good except some frequency points. To the best of our knowledge, not many examples of FRFs in centrifuge dynamic testing as shown in Fig. 9 have appeared in literature. It may be seen that there is a peak at around $20 \mathrm{~Hz}$. This peak corresponds 


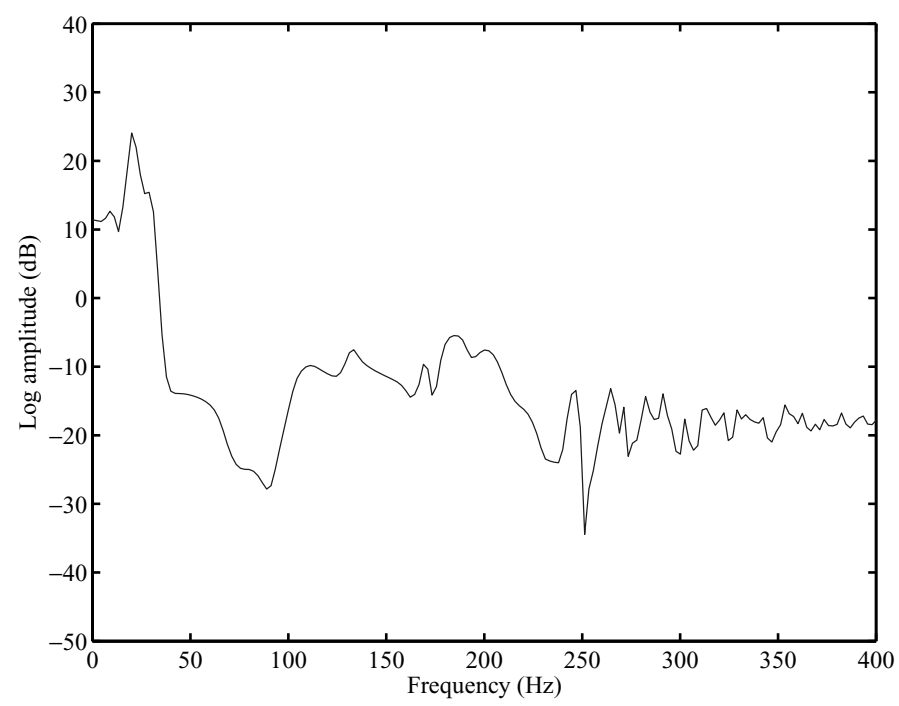

(a) Frequency response function

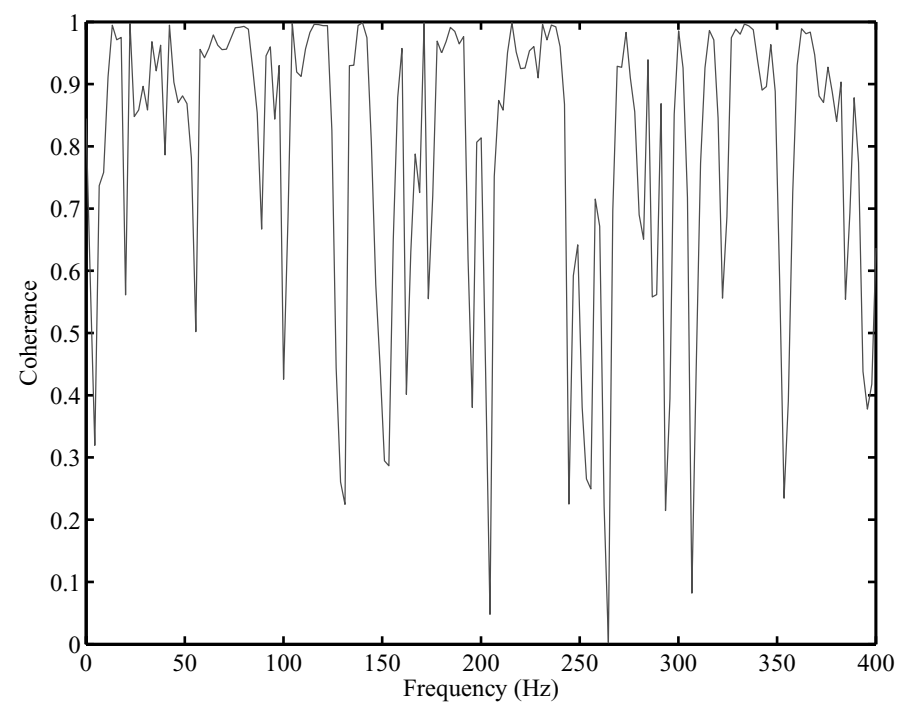

(b) Coherence

Fig. 9. Frequency response function and coherence between the input to the pile and the output of the pile head.

to the first natural frequency of the combines system so that $f_{1}=20 \mathrm{~Hz}$ (see reference [3] for the detailed frequency domain analysis). As the input motion is predominantly harmonic and if we assume linear (between force and displacement) SDOF system, then this peak would correspond to the natural frequency of the oscillator. In the next section we investigate this in more details using analytical means.

\section{Analytical formulation}

\subsection{Equation of motion and boundary conditions}

We consider an Euler Bernoulli beam as shown in Fig. 10. The bending stiffness of the beam is $E I$ and it is resting against a linear uniform elastic support of stiffness $k$. The beam has a tip mass with rotary inertia $J$ and mass 


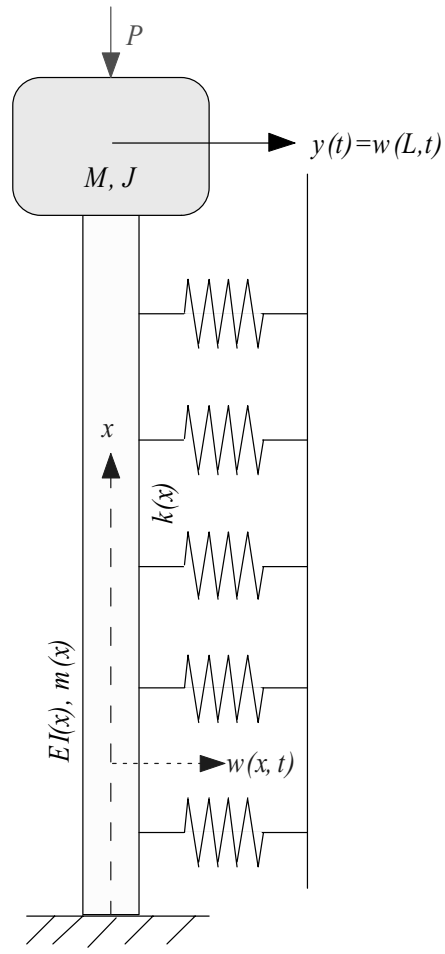

Fig. 10. Combined pile-soil model using Euler Bernoulli beam with tip mass and axial force resting against a distributed elastic support.

$M$. The mass per unit length of the beam is $m, r$ is the radius of gyration and the beam is subjected to a constant compressive axial load $P$. The equation of motion of the beam is given by

$$
\begin{aligned}
& \frac{\partial^{2}}{\partial x^{2}}\left(E I(x) \frac{\partial^{2} w(x, t)}{\partial x^{2}}\right)+\frac{\partial}{\partial x}\left(P(x) \frac{\partial w(x, t)}{\partial x}\right)-\frac{\partial}{\partial x}\left(m r^{2}(x) \frac{\partial \ddot{w}(x, t)}{\partial x}\right) \\
& \quad+k(x) w(x, t)+m \ddot{w}(x, t)=f(x, t) .
\end{aligned}
$$

here $w(x, t)$ is the transverse deflection of the beam, $x$ is the spatial coordinate along the length of the beam, $t$ is time, $(\bullet)$ denotes derivative with respect to time and $f(x, t)$ is the applied time depended load on the beam. It is assumed that all properties are constant along the length of the beam. Equation (1) is a fourth-order partial differential equation [24] and has been treated extensively in literature (see for example, references $[1,9-13,15-22$, $25,29,30,36,38-40])$. Our central aim is to obtain the natural frequency of the system. The book by Blevins [8] lists several expressions of the natural frequencies of similar systems but this particular case has not been covered. Here we develop an approach based on the non-dimensionalisation of the equation of motion in Fig. 10.

Because we are interested in the free vibration problem, considering $f(x, t)=0$, Eq. (1) can be expressed as

$$
E I \frac{\partial^{4} w(x, t)}{\partial x^{4}}+P \frac{\partial^{2} w(x, t)}{\partial x^{2}}-m r^{2} \frac{\partial^{2} \ddot{w}(x, t)}{\partial x^{2}}+k w(x, t)+m \ddot{w}(x, t)=0 .
$$

The four boundary conditions associated with this equation can be expressed as

- Deflection at $x=0$ :

$$
w(0, t)=0 .
$$

- Rotation at $x=0$ :

$$
\frac{\partial w(x, t)}{\partial x}=\left.0\right|_{x=0} \text { or } w^{\prime}(0, t)=0 .
$$


- Bending moment at $x=L$ :

$$
E I \frac{\partial^{2} w(x, t)}{\partial x^{2}}+J \frac{\partial \ddot{w}(x, t)}{\partial x}=\left.0\right|_{x=L} \text { or } E I w^{\prime \prime}(L, t)+J \frac{\partial \ddot{w}(L, t)}{\partial x}=0 .
$$

- Shear force at $x=L$ :

$$
\begin{aligned}
& E I \frac{\partial^{3} w(x, t)}{\partial x^{3}}+P \frac{\partial w(x, t)}{\partial x}-M \ddot{w}(x, t)-m r^{2} \frac{\partial \ddot{w}(x, t)}{\partial x}=\left.0\right|_{x=L} \\
& \text { or } E I W^{\prime \prime \prime}(L, t)+P w^{\prime}(L, t)-M \ddot{w}(L, t)-m r^{2} \frac{\partial \ddot{w}(L, t)}{\partial x}=0
\end{aligned}
$$

Assuming harmonic solution (the separation of variable) we have

$$
w(x, t)=W(\xi) \exp \{i \omega t\}, \quad \xi=x / L .
$$

Substituting this in the equation of motion and the boundary conditions, Eqs (2)-(6), results

$$
\begin{aligned}
& \frac{E I}{L^{4}} \frac{\partial^{4} W(\xi)}{\partial \xi^{4}}+\frac{P}{L^{2}} \frac{\partial^{2} W(\xi)}{\partial \xi^{2}}+k W(\xi)-m w^{2} W(\xi)+\frac{m r^{2} w^{2}}{L^{2}} \frac{\partial^{2} W(\xi)}{\partial \xi^{2}}=0 \\
& W(0)=0 \\
& W^{\prime}(0)=0 \\
& \frac{E I}{L^{2}} W^{\prime \prime}(1)-\frac{\omega^{2} J}{L} W^{\prime}(1)=0 \\
& \frac{E I}{L^{3}} W^{\prime \prime \prime}(1)+\frac{P}{L} W^{\prime}(1)+\omega^{2} M W(1)+\frac{m r^{2} \omega^{2}}{L} W^{\prime}(1)=0 .
\end{aligned}
$$

It is convenient to express these equations in terms of non-dimensional parameters by elementary rearrangements as

$$
\frac{\partial^{4} W(\xi)}{\partial \xi^{4}}+\tilde{v} \frac{\partial^{2} W(\xi)}{\partial \xi^{2}}+\eta W(\xi)-\Omega^{2} W(\xi)=0
$$

$$
W(0)=0
$$

$W^{\prime}(0)=0$

$$
W^{\prime \prime}(1)-\beta \Omega^{2} W^{\prime}(1)=0
$$

$W^{\prime \prime \prime}(1)+\widetilde{\nu} W^{\prime}(1)+\alpha \Omega^{2} W(1)=0$

where

$$
\widetilde{\nu}=\nu+\mu^{2} \Omega^{2}
$$

and

$$
\begin{aligned}
& \nu=\frac{P L^{2}}{E I} \quad(\text { nondimensional axial force }) \\
& \eta=\frac{k L^{4}}{E I} \quad(\text { nondimensional support stiffness }) \\
& \Omega^{2}=\omega^{2} \frac{m L^{4}}{E I} \quad \text { (nondimensional frequency parameter) }
\end{aligned}
$$




$$
\begin{aligned}
& \alpha=\frac{M}{m L} \quad \text { (mass ratio) } \\
& \beta=\frac{J}{m L^{3}} \quad \text { (nondimensional rotary inertia) } \\
& \mu=\frac{r}{L} \quad \text { (nondimensional radius of gyration). }
\end{aligned}
$$

It is often useful to express $\nu$ in terms of the critical buckling load as

$$
\nu=\frac{\pi^{2}}{4}\left(P / P_{c r}\right)=\frac{\pi^{2}}{4} \theta_{c}
$$

where

$$
\theta_{c}=P / P_{c r}
$$

is the ratio between the applied load and the critical buckling load. Also note that for most beams $\mu=r / L \ll 1$ so that $\mu^{2} \approx 0$. As a result for low frequency vibration one expects $\widetilde{\nu} \approx \nu$. For notational convenience we define the natural frequency scaling parameter

$$
f_{0}=\sqrt{\frac{E I}{m L^{4}}} .
$$

Using this, from Eq. (21) the natural frequencies of the system can be obtained as

$$
\omega_{j}=\Omega_{j} f_{0} ; \quad j=1,2,3, \cdots
$$

\subsection{Derivation of the frequency equations}

Assuming a solution of the form

$$
W(\xi)=\exp \{\lambda \xi\}
$$

and substituting in the equation of motion Eq. (13) results

$$
\lambda^{4}+\widetilde{\nu} \lambda^{2}-\left(\Omega^{2}-\eta\right)=0
$$

This equation is often know as the dispersion relationship. This is the equation governing the natural frequencies of the beam. Solving this equation for $\lambda^{2}$ we have

$$
\begin{aligned}
\lambda^{2} & =-\frac{\widetilde{\nu}}{2} \pm \sqrt{\left(\frac{\widetilde{\nu}}{2}\right)^{2}+\left(\Omega^{2}-\eta\right)} \\
& =-\left(\sqrt{\left(\frac{\widetilde{\nu}}{2}\right)^{2}+\left(\Omega^{2}-\eta\right)}+\frac{\widetilde{\nu}}{2}\right), \quad\left(\sqrt{\left(\frac{\widetilde{\nu}}{2}\right)^{2}+\left(\Omega^{2}-\eta\right)}-\frac{\widetilde{\nu}}{2}\right) .
\end{aligned}
$$

Depending on whether $\Omega^{2}-\eta>0$ or not two cases arise.

Case 1: If $\widetilde{\nu}>0$ and $\Omega^{2}-\eta>0$ or $\Omega^{2}>\eta$ then both roots are real with one negative and one positive root. Therefore, the four roots can be expressed as

$$
\lambda= \pm i \lambda_{1}, \quad \pm \lambda_{2}
$$

where

$$
\lambda_{1}=\left(\sqrt{\left(\frac{\widetilde{\nu}}{2}\right)^{2}+\left(\Omega^{2}-\eta\right)}+\frac{\widetilde{\nu}}{2}\right)^{1 / 2}
$$


and $\lambda_{2}=\left(\sqrt{\left(\frac{\widetilde{\nu}}{2}\right)^{2}+\left(\Omega^{2}-\eta\right)}-\frac{\widetilde{\nu}}{2}\right)^{1 / 2}$.

From Eqs (33) and (34) also note that

$$
\lambda_{1}^{2}-\lambda_{2}^{2}=\widetilde{\nu} .
$$

In view of the roots in in Eq. (32) the solution $W(\xi)$ can be expressed as

$W(\xi)=a_{1} \sin \lambda_{1} \xi+a_{2} \cos \lambda_{1} \xi+a_{3} \sinh \lambda_{2} \xi+a_{4} \cosh \lambda_{2} \xi$ or $W(\xi)=\mathbf{s}^{T}(\xi) \mathbf{a}$

where the vectors

$$
\mathbf{s}(\xi)=\left\{\sin \lambda_{1} \xi, \cos \lambda_{1} \xi, \sinh \lambda_{2} \xi, \cosh \lambda_{2} \xi\right\}^{T}
$$

and $\mathbf{a}=\left\{a_{1}, a_{2}, a_{3}, a_{4}\right\}^{T}$.

Applying the boundary conditions in Eqs (14)-(17) on the expression of $W(\xi)$ in Eq. (36) we have

$$
\mathbf{R a}=\mathbf{0}
$$

where the matrix

$$
\mathbf{R}=\left[\begin{array}{cc}
s_{1}(0) & s_{2}(0) \\
s_{1}^{\prime}(0) & s_{2}^{\prime}(0) \\
s_{1}^{\prime \prime}(1)-\beta \Omega^{2} s_{1}^{\prime}(1) & s_{2}^{\prime \prime}(1)-\beta \Omega^{2} s_{2}^{\prime}(1) \\
s_{1}^{\prime \prime \prime}(1)+\widetilde{\nu} s_{1}^{\prime}(1)+\alpha \Omega^{2} s_{1}(1) & s_{2}^{\prime \prime \prime}(1)+\widetilde{\nu} s_{2}^{\prime}(1)+\alpha \Omega^{2} s_{2}(1) \\
s_{3}(0) & s_{4}(0) \\
s_{3}^{\prime}(0) & s_{4}^{\prime}(0) \\
s_{3}^{\prime \prime}(1)-\beta \Omega^{2} s_{3}^{\prime}(1) & s_{4}^{\prime \prime}(1)-\beta \Omega^{2} s_{4}^{\prime}(1) \\
s_{3}^{\prime \prime \prime}(1)+\widetilde{\nu} s_{3}^{\prime}(1)+\alpha \Omega^{2} s_{3}(1) & s_{3}^{\prime \prime \prime}(1)+\widetilde{\nu} s_{3}^{\prime}(1)+\alpha \Omega^{2} s_{3}(1)
\end{array}\right] .
$$

Substituting functions $s_{j}(\xi), j=1, \cdots, 4$ from Eq. (37) and simplifying we obtain

$$
\mathbf{R}=\left[\begin{array}{cc}
0 & 1 \\
\lambda_{1} & 0 \\
-\sin \left(\lambda_{1}\right) \lambda_{1}{ }^{2}-\Omega^{2} \beta \cos \left(\lambda_{1}\right) \lambda_{1} & -\cos \left(\lambda_{1}\right) \lambda_{1}{ }^{2}+\Omega^{2} \beta \sin \left(\lambda_{1}\right) \lambda_{1} \\
0 & 1 \\
\lambda_{2} & 0 \\
\cos \left(\lambda_{1}\right) \lambda_{1}{ }^{3}+\widetilde{\nu} \cos \left(\lambda_{1}\right) \lambda_{1}+\Omega^{2} \alpha \sin \left(\lambda_{1}\right) \sin \left(\lambda_{1}\right) \lambda_{1}{ }^{3}-\widetilde{\nu} \sin \left(\lambda_{1}\right) \lambda_{1}+\Omega^{2} \alpha \cos \left(\lambda_{1}\right) \\
\sinh \left(\lambda_{2}\right) \lambda_{2}{ }^{2}-\Omega^{2} \beta \cosh \left(\lambda_{2}\right) \lambda_{2} & \cosh \left(\lambda_{2}\right) \lambda_{2}{ }^{2}-\Omega^{2} \beta \sinh \left(\lambda_{2}\right) \lambda_{2} \\
\cosh \left(\lambda_{2}\right) \lambda_{2}{ }^{3}+\widetilde{\nu} \cosh \left(\lambda_{2}\right) \lambda_{2}+\Omega^{2} \alpha \sinh \left(\lambda_{2}\right) \sinh \left(\lambda_{2}\right) \lambda_{2}{ }^{3}+\widetilde{\nu} \sinh \left(\lambda_{2}\right) \lambda_{2}+\Omega^{2} \alpha \cosh \left(\lambda_{2}\right)
\end{array}\right] .
$$

The constant vector in Eq. (39) cannot be zero. Therefore, the equation governing the natural frequencies is given by

$|\mathbf{R}|=0$.

This, upon simplification (see Appendix A for the Maple ${ }^{\circledR}$ code developed for this purpose) reduces to

$$
\begin{aligned}
& \left(-\sin \left(\lambda_{1}\right) \lambda_{1}{ }^{2} \lambda_{2} \Omega^{2} \cosh \left(\lambda_{2}\right)+\lambda_{1} \Omega^{2} \cos \left(\lambda_{1}\right) \sinh \left(\lambda_{2}\right) \lambda_{2}{ }^{2}-\Omega^{4} \beta \sin \left(\lambda_{1}\right) \lambda_{1}{ }^{2} \sinh \left(\lambda_{2}\right)\right. \\
& -\Omega^{2} \sin \left(\lambda_{1}\right) \cosh \left(\lambda_{2}\right) \lambda_{2}{ }^{3}+\Omega^{4} \sin \left(\lambda_{1}\right) \beta \sinh \left(\lambda_{2}\right) \lambda_{2}{ }^{2}+\cos \left(\lambda_{1}\right) \lambda_{1}{ }^{3} \Omega^{2} \sinh \left(\lambda_{2}\right) \\
& \left.-2 \lambda_{1} \Omega^{4} \cos \left(\lambda_{1}\right) \beta \cosh \left(\lambda_{2}\right) \lambda_{2}+2 \Omega^{4} \lambda_{2} \beta \lambda_{1}\right) \alpha+\left(\lambda_{1} \lambda_{2}{ }^{3}-\cos \left(\lambda_{1}\right) \lambda_{1} \cosh \left(\lambda_{2}\right) \lambda_{2}{ }^{3}\right. \\
& \left.-2 \sin \left(\lambda_{1}\right) \lambda_{1}{ }^{2} \sinh \left(\lambda_{2}\right) \lambda_{2}{ }^{2}-\lambda_{1}{ }^{3} \lambda_{2}+\cos \left(\lambda_{1}\right) \lambda_{1}{ }^{3} \cosh \left(\lambda_{2}\right) \lambda_{2}\right) \widetilde{\nu}+\lambda_{1}{ }^{5} \lambda_{2}+\lambda_{1} \lambda_{2}{ }^{5} \\
& +2 \cos \left(\lambda_{1}\right) \lambda_{1}{ }^{3} \cosh \left(\lambda_{2}\right) \lambda_{2}{ }^{3}+\sin \left(\lambda_{1}\right) \lambda_{1}{ }^{4} \sinh \left(\lambda_{2}\right) \lambda_{2}{ }^{2}-\sin \left(\lambda_{1}\right) \lambda_{1}{ }^{2} \sinh \left(\lambda_{2}\right) \lambda_{2}{ }^{4} \\
& -\sin \left(\lambda_{1}\right) \lambda_{1}{ }^{4} \Omega^{2} \beta \cosh \left(\lambda_{2}\right) \lambda_{2}-\Omega^{2} \beta \sin \left(\lambda_{1}\right) \lambda_{1}{ }^{2} \cosh \left(\lambda_{2}\right) \lambda_{2}{ }^{3}-\Omega^{2} \beta \cos \left(\lambda_{1}\right) \lambda_{1} \sinh \left(\lambda_{2}\right) \lambda_{2}{ }^{4} \\
& -\cos \left(\lambda_{1}\right) \lambda_{1}{ }^{3} \Omega^{2} \beta \sinh \left(\lambda_{2}\right) \lambda_{2}{ }^{2}=0 .
\end{aligned}
$$


The natural frequencies can be obtained by solving Eq. (43) for $\Omega$. Due to the complexity of this transcendental equation it should be solved numerically.

Case 2: If $\widetilde{\nu}>0$ and $\Omega^{2}-\eta<0$ or $\Omega^{2}<\eta$ then both the roots are real and negative. Therefore, all of the four roots can be expressed as

$$
\lambda= \pm i \lambda_{1}, \quad \pm i \widehat{\lambda}_{2}
$$

where $\lambda_{1}$ is as in the previous case

$$
\lambda_{1}=\left(\frac{\widetilde{\nu}}{2}+\sqrt{\left(\frac{\widetilde{\nu}}{2}\right)^{2}-\left(\eta-\Omega^{2}\right)}\right)^{1 / 2}
$$

and $\widehat{\lambda}_{2}$ is given by

$$
\widehat{\lambda}_{2}=\left(\frac{\widetilde{\nu}}{2}-\sqrt{\left(\frac{\widetilde{\nu}}{2}\right)^{2}-\left(\eta-\Omega^{2}\right)}\right)^{1 / 2} .
$$

In view of the roots in in Eq. (44) the solution $W(\xi)$ can be expressed as

$$
W(\xi)=a_{1} \sin \lambda_{1} \xi+a_{2} \cos \lambda_{1} \xi+a_{3} \sin \widehat{\lambda}_{2} \xi+a_{4} \cos \widehat{\lambda}_{2} \xi \text { or } W(\xi)=\mathbf{s}^{T}(\xi) \mathbf{a}
$$

where the vectors

$$
\begin{aligned}
& \mathbf{s}(\xi)=\left\{\sin \lambda_{1} \xi, \cos \lambda_{1} \xi, \sin \widehat{\lambda}_{2} \xi, \cos \widehat{\lambda}_{2} \xi\right\}^{T} \\
& \mathbf{a}=\left\{a_{1}, a_{2}, a_{3}, a_{4}\right\}^{T} .
\end{aligned}
$$

Applying the boundary conditions in Eqs (14)-(17) on the expression of $W(\xi)$ in Eq. (47)and following similar procedure as the previous case (see Appendix A for the Maple ${ }^{\circledR}$ code developed for this purpose), the frequency equation can be expressed as

$$
\begin{aligned}
& \left(\cos \left(\lambda_{1}\right) \lambda_{1} \cos \left(\widehat{\lambda}_{2}\right) \hat{\lambda}_{2}^{3}-\lambda_{1} \widehat{\lambda}_{2}^{3}-\lambda_{1}{ }^{3} \widehat{\lambda}_{2}+\cos \left(\lambda_{1}\right) \lambda_{1}{ }^{3} \cos \left(\widehat{\lambda}_{2}\right) \lambda_{2}+2 \sin \left(\lambda_{1}\right) \lambda_{1}{ }^{2} \sin \left(\widehat{\lambda}_{2}\right) \widehat{\lambda}_{2}^{2}\right) \widetilde{\nu} \\
& +\left(-\Omega^{4} \beta \sin \left(\lambda_{1}\right) \lambda_{1}{ }^{2} \sin \left(\widehat{\lambda}_{2}\right)+\cos \left(\lambda_{1}\right) \lambda_{1}^{3} \Omega^{2} \sin \left(\widehat{\lambda}_{2}\right)-2 \lambda_{1} \Omega^{4} \cos \left(\lambda_{1}\right) \beta \cos \left(\hat{\lambda}_{2}\right) \hat{\lambda}_{2}+2 \lambda_{1} \Omega^{4} \beta \widehat{\lambda}_{2}\right. \\
& -\lambda_{1} \Omega^{2} \cos \left(\lambda_{1}\right) \sin \left(\widehat{\lambda}_{2}\right) \hat{\lambda}_{2}^{2}+\Omega^{2} \sin \left(\lambda_{1}\right) \cos \left(\widehat{\lambda}_{2}\right) \hat{\lambda}_{2}^{3}-\sin \left(\lambda_{1}\right) \lambda_{1}{ }^{2} \widehat{\lambda}_{2} \Omega^{2} \cos \left(\widehat{\lambda}_{2}\right) \\
& \left.-\Omega^{4} \sin \left(\lambda_{1}\right) \beta \sin \left(\hat{\lambda}_{2}\right) \hat{\lambda}_{2}^{2}\right) \alpha-2 \cos \left(\lambda_{1}\right) \lambda_{1}^{3} \cos \left(\hat{\lambda}_{2}\right) \hat{\lambda}_{2}^{3}-\sin \left(\lambda_{1}\right) \lambda_{1}{ }^{4} \sin \left(\widehat{\lambda}_{2}\right) \hat{\lambda}_{2}^{2} \\
& -\sin \left(\lambda_{1}\right) \lambda_{1}{ }^{2} \sin \left(\hat{\lambda}_{2}\right) \hat{\lambda}_{2}^{4}+\lambda_{1}{ }^{5} \widehat{\lambda}_{2}-\Omega^{2} \beta \cos \left(\lambda_{1}\right) \lambda_{1} \sin \left(\widehat{\lambda}_{2}\right) \hat{\lambda}_{2}^{4}+\Omega^{2} \beta \sin \left(\lambda_{1}\right) \lambda_{1}{ }^{2} \cos \left(\widehat{\lambda}_{2}\right) \hat{\lambda}_{2}^{3} \\
& -\sin \left(\lambda_{1}\right) \lambda_{1}^{4} \Omega^{2} \beta \cos \left(\widehat{\lambda}_{2}\right) \hat{\lambda}_{2}+\cos \left(\lambda_{1}\right) \lambda_{1}{ }^{3} \Omega^{2} \beta \sin \left(\lambda_{2}\right) \widehat{\lambda}_{2}^{2}+\lambda_{1} \widehat{\lambda}_{2}^{5}=0 .
\end{aligned}
$$

The natural frequencies can be obtained by solving Eq. (50) for $\Omega$. Due to the complexity of this transcendental equation it should be solved numerically.

\subsection{Special cases}

Equations (43) and (50) are quite general as they consider axial load, elastic support, tip mass, and rotary inertia. Several interesting special cases discussed in literature can be obtained from these expressions. 
- Standard cantilever: no tip mass, rotary inertia, axial force and support stiffness:

For this case $\eta=0, \beta=0, \alpha=0$ and $\nu=0$. From the dispersion relationship in Eq. (30) observe that for this case $\lambda_{1}=\lambda_{2}=\sqrt{\Omega}=\omega \sqrt{\frac{m L^{4}}{E I}}=\lambda$ (say). As a result, the two cases discussed before converge to a single case. Substituting these in Eq. (43) or (50) and simplifying (see the Maple ${ }^{\circledR}$ script in Appendix ) we obtain the frequency equation as

$$
1+\cos (\lambda) \cosh (\lambda)=0 .
$$

This matches exactly with the frequency equation for a standard cantilever (see the book by Meirovitch [27] or Géradin and Rixen [14]).

- Cantilever with a tip mass: no rotary inertia, axial force and support stiffness:

For this case $\eta=0, \beta=0$, and $\nu=0$. From the dispersion relationship in Eq. (30) observe that for this case again $\lambda_{1}=\lambda_{2}=\lambda$ (say). Substituting these in Eq. (43) or (50) and simplifying (see the Maple ${ }^{\circledR}$ script in Appendix A) we obtain the frequency equation as

$$
(-\sin (\lambda) \cosh (\lambda)+\cos (\lambda) \sinh (\lambda)) \alpha \lambda+\cos (\lambda) \cosh (\lambda)+1=0 .
$$

If we substitute $\alpha=0$ in this equation, we retrieve the standard cantilever case obtained in Eq. (51).

- Cantilever with a tip mass and rotary inertia: no axial force and support stiffness:

For this case only $\eta=0$ and $\nu=0$ and we also have $\lambda_{1}=\lambda_{2}=\lambda$ (say). Substituting these in Eq. (43) or (50) and simplifying (see the Maple ${ }^{\circledR}$ script in Appendix A) we obtain the frequency equation as

$$
\begin{gathered}
(-\cos (\lambda) \cosh (\lambda)+1) \alpha \beta \lambda^{4}+(-\cos (\lambda) \sinh (\lambda)-\sin (\lambda) \cosh (\lambda)) \beta \lambda^{3} \\
+(-\sin (\lambda) \cosh (\lambda)+\cos (\lambda) \sinh (\lambda)) \alpha \lambda+\cos (\lambda) \cosh (\lambda)+1=0 .
\end{gathered}
$$

If we substitute $\beta=0$ in this equation, we retrieve the case obtained in Eq. (52).

\section{Numerical results}

In this section we aim to compare experimental results presented in subsection 3.3 with the analytical expressions developed in the last section. First we determine the relevant non-dimensional parameters for the experiment appearing in the equations derived here. We focus our attention on the affect of $\theta_{c}=P / P_{c r}$ ratio and the nondimensional soil stiffness $\eta$ on the first-natural frequency. For this reason, the numerical results are presented as a function of $\theta_{c}$ and $\eta$.

Recall that the centrifuge tests were conducted at $50 \mathrm{~g}$ acceleration. Therefore the non-dimensional mass ratio can be obtained as

$$
\alpha=\frac{M}{m L}=\frac{P}{50 \mathrm{gmL}}=\frac{P}{P_{c r}}\left(\frac{P_{c r}}{50 \mathrm{gmL}}\right)=\frac{P}{P_{c r}}\left(\frac{\pi^{2} E I}{200 \mathrm{gmL}^{3}}\right)=\theta_{c}\left(\frac{\pi^{2} f_{0}^{2} \mathrm{~L}}{200 \mathrm{~g}}\right) .
$$

The mass at the top of the beam used in the experiment is of cylindrical shape. Supposing its height is $h$ and radius is $a$, the moment of inertia can be obtained as

$$
J=\frac{M}{12}\left(3 a^{2}+h^{2}\right) .
$$

Therefore, the nondimensional rotary inertia can be obtained as

$$
\beta=\frac{J}{m L^{3}}=\frac{M\left(3 a^{2}+h^{2}\right)}{12 \mathrm{~mL}^{3}}=\frac{M}{m L}\left[\frac{1}{4}\left(\frac{a}{L}\right)^{2}+\frac{1}{12}\left(\frac{h}{L}\right)^{2}\right]=\alpha\left(\phi_{a}^{2} / 4+\phi_{h}^{2} / 12\right) .
$$

In the context of pile supported buildings, the nondimensional radius $\phi_{a}$ and the nondimensional height $\phi_{h}$ can be considered as the 'shape parameters' of the building. These parameters take account of the physical shape of the building so that it is not considered as a 'point mass' used in many simplified analyses. For non-circular masses (buildings) $a$ may replaced by the radius of gyration about the $z$-axis (vertical axis). For the experimental study (see 
Table 2) $E I=7.77 \times 10^{6} \mathrm{~N} \mathrm{~mm}^{2}, L=189 \mathrm{~mm}$ and $m=0.3 \mathrm{gm} / \mathrm{mm}$. Therefore the natural frequency scaling parameter can be obtained as

$$
f_{0}=\frac{E I}{m L^{4}}=145.86 \mathrm{~s}^{-1} .
$$

The radius and the height of the mass are respectively $a=34 \mathrm{~mm}$ and $h=18 \mathrm{~mm}$. Using these values from Eqs (54) and (56) we obtain

$$
\alpha=20.233 \theta_{c} \text { and } \beta=0.009 \alpha=0.1821 \theta_{c} .
$$

The radius of gyration of the pile (the outside diameter is $9.3 \mathrm{~mm}$ and the inside diameter is $8.5 \mathrm{~mm}$ ) is $3.1 \mathrm{~mm}$ so that the nondimensional radius of gyration $\mu=r / L=0.016$. From Eq. (18) we therefore have

$$
\widetilde{\nu}=\nu+2.56 \times 10^{-4} \Omega^{2} .
$$

For $40 \%$ relative density, modulus of subgrade reaction is $8 \mathrm{MN} / \mathrm{m}^{3}$ (see reference [3] for further details). It is often convenient to calculate the parameters at the prototype scale and use the scaling laws. The experiment being carried out at $50 \mathrm{~g}$, and therefore the prototype stiffness of the pile-soil system $(k)$ is $8 \mathrm{MN} / \mathrm{m}^{3} \times 0.465 \mathrm{~m}=$ 3.72 MPa. Based on this value we have

$$
\eta=\frac{k L^{4}}{E I}=610.10 \text {. }
$$

During the full liquefaction, the value $k$ reduces to less than $10 \%$ of the original value [31].

Depending on the two cases discussed before, we substitute these values of $\eta$ and $\widetilde{\nu}$ either in Eqs (33) and (34) to obtain $\lambda_{1}$ and $\lambda_{2}$, or in Eqs (45) and (46) to obtain $\lambda_{1}$ and $\widehat{\lambda}_{2}$. Substituting them either in Eq. (43) or (50) we solve for the nondimensional first natural frequency $\Omega_{1}$. For earthquake applications the first natural frequency is the most important as the excitation frequency is generally between 1-10 Hz. Higher natural frequencies can however be obtained by solving Eq. (43) or (50).

The variation of the first natural frequency of the cantilever pile with respect to the axial load for different values of normalized soil stiffness is shown in Fig. 11. From Fig. 9(a) it can be observed that (see reference [3] for the detailed analysis) the first natural frequency is $20 \mathrm{~Hz}$. At $50 \mathrm{~g}$ acceleration, the $P / P_{c r}$ ratio becomes 0.5 . Using these two values, from Fig. 11 we observe that $\eta \approx 35$, which about $5.75 \%$ of the original value. Experiment shows that the first natural frequency is $20 \mathrm{~Hz}\left(f_{1}=19.42 \mathrm{~Hz}\right.$ to be precise) at the full liquefaction. Here $\omega=2 \pi f$, where $f$ in the frequency in Hz. Our analytical model suggests that at $20 \mathrm{~Hz}$ is reached when the soil stiffness is $0.06 \%$ of the initial value, which is comparable to the definition of liquefaction based on AIJ [31]. In Fig. 11 we have plotted the experimental data (marked by '*').

The variation of the first natural frequency of the cantilever pile with respect to the normalized support stiffness for different values of axial load is shown in Fig. 12. In the same diagram the experimental result is shown by a '*'. This plot confirms that when $\eta=35.0$, then $P / P_{c r}=0.5$ for the experimentally measured first natural frequency of $20 \mathrm{~Hz}$.

In Fig. 13, the overall variation of the first natural frequency of the cantilever pile with respect to both normalized support stiffness and axial load are shown in a 3D plot. The interesting feature to observe from this plot is the rapid and sharp 'fall' in the natural frequency for small values of $\eta$. This has very important practical consequence. This result implies that the system natural frequency can come very close to the earthquake excitation frequency $(\approx 1-10 \mathrm{~Hz})$ when soils is approaching full liquefaction than previously thought. Also observe that the reduction in the first natural frequency become even sharper for higher values of $P / P_{c r}$. These nonlinear effects must be taken into account for earthquake resistant design of pile foundations.

\section{Conclusions}

Dynamic instability of pile-supported structures founded on liquefiable soils has been investigated. Small-scale model tests and analytical work form the basis of this investigation. Three conditions are studied, namely (a) the soil supporting the foundation is stiff which may represent the condition prior to the earthquake, (b) the soil supporting 


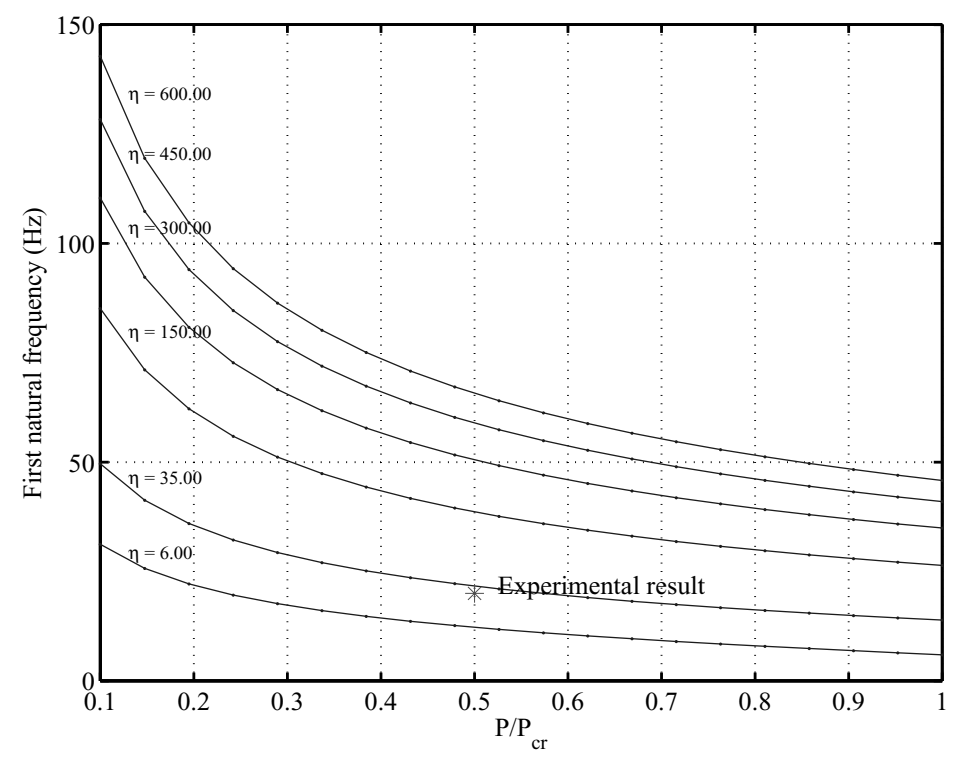

Fig. 11. Variation of the first natural frequency of the cantilever pile with respect to the axial load for different values of normalized soil stiffness. The experimental result ( $f_{1}=20 \mathrm{~Hz}, \theta_{c}=P / P_{c r}=0.5$ and $\eta=35.0$ ) is shown by a '*' in the diagram.

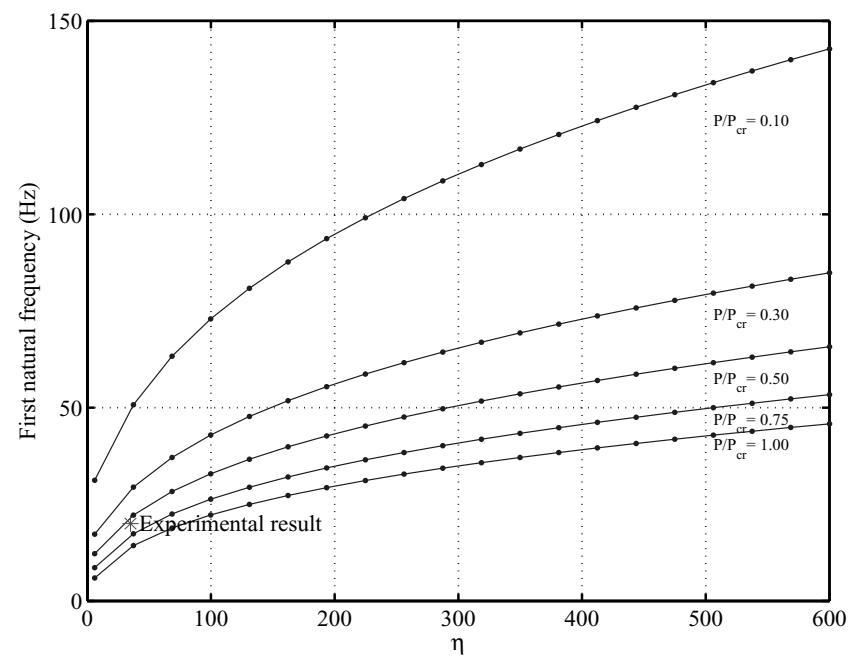

Fig. 12. Variation of the first natural frequency of the cantilever pile with respect to the normalized support stiffness for different values of axial load. The experimental result $\left(f_{1}=20 \mathrm{~Hz}, \theta_{c}=P / P_{c r}=0.5\right.$ and $\left.\eta=35.0\right)$ is shown by a '*' in the diagram.

the foundation is fully liquefied (zero stiffness) which may represent some time instant after the onset of the shaking, and (c) the transient phase when the supporting soil is being transformed from being stiff to being liquefied.

The experimental results indicate that the first natural frequency of the structure reduces as the soil starts to lose its stiffness (transient phase) and is lowest when the soil is in the fully liquefied condition. Bending, buckling and dynamics should be considered simultaneously to analytically model the real system. A distributed parameter model using the Euler Bernoulli beam theory with axial load, support stiffness and tip mass with rotary inertia is considered. The non-dimensional parameters necessary to understand the dynamic instability have been identified. These parameters are nondimensional axial force $(\nu)$, nondimensional soil stiffness, $(\eta)$, mass ratio between the building and the pile $(\alpha)$, nondimensional radius of gyration of the pile $(\mu)$, ratio between the applied load and the critical buckling load $\left(\theta_{c}\right)$, and the shape parameters of the building, namely the nondimensional radius $\left(\phi_{a}\right)$ and the 


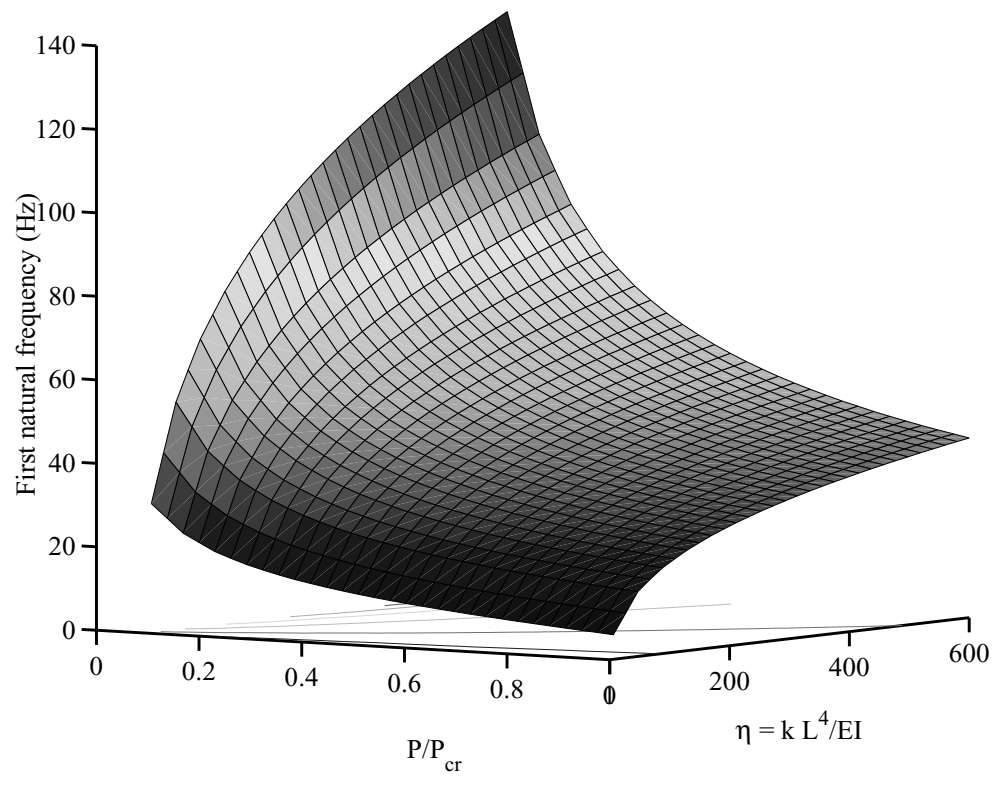

Fig. 13. Variation of the first natural frequency of the cantilever pile with respect to the normalized support stiffness and axial load.

nondimensional height $\left(\phi_{h}\right)$. The shape parameters take account of the physical shape of the building so that it is not considered as a point mass used in many simplified analyses.

Current codes of practice for piled foundations such as the Japanese code and the Eurocode are based only on the bending criteria where the lateral loads induce bending stress in the pile. It is well recognized that codes of practice have to specify some simplified approach for design which should provide a safe working envelope for any structure of the class being considered, and in full range of ground conditions likely to be encountered at different sites. Our concern in this paper is to point out that the application of such simplified approach demands further consideration of effective lateral stiffness of the pile taking into account the change in the natural frequency during liquefaction. One of the key conclusion is that the first natural frequency of the foundation will start to drop when the soil transforms from being solid to liquid. If the first natural frequency comes close to the excitation frequency of the earthquake motion (typically between $0.5 \mathrm{~Hz}$ and $10 \mathrm{~Hz}$ ), then the amplitude of vibration (and consequently the internal stresses) can grow significantly. Using the expressions derived in the paper, designers could estimate the first natural frequency at the full liquefaction and design the pile such that the resulting natural frequency does not come close to the expected frequency of the earthquake motion.

\section{Maple $^{\circledR}$ Code for the frequency equations}

Here we show the code in Maple ${ }^{\circledR}[28]$ used to generate the equations for the natural frequencies, that is Eqs (43) and (50). Codes used to obtain the special cases discussed in subsection 4.3 are also given below.

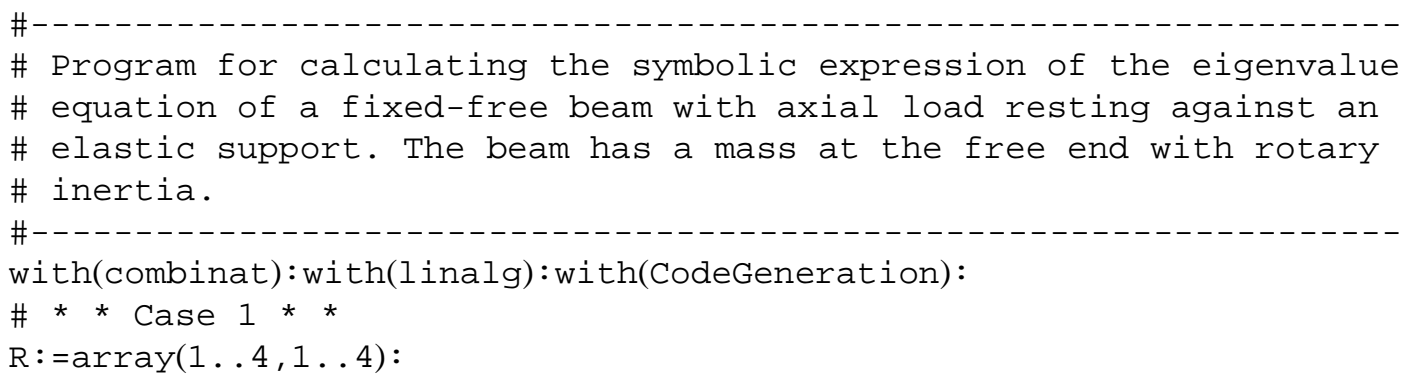




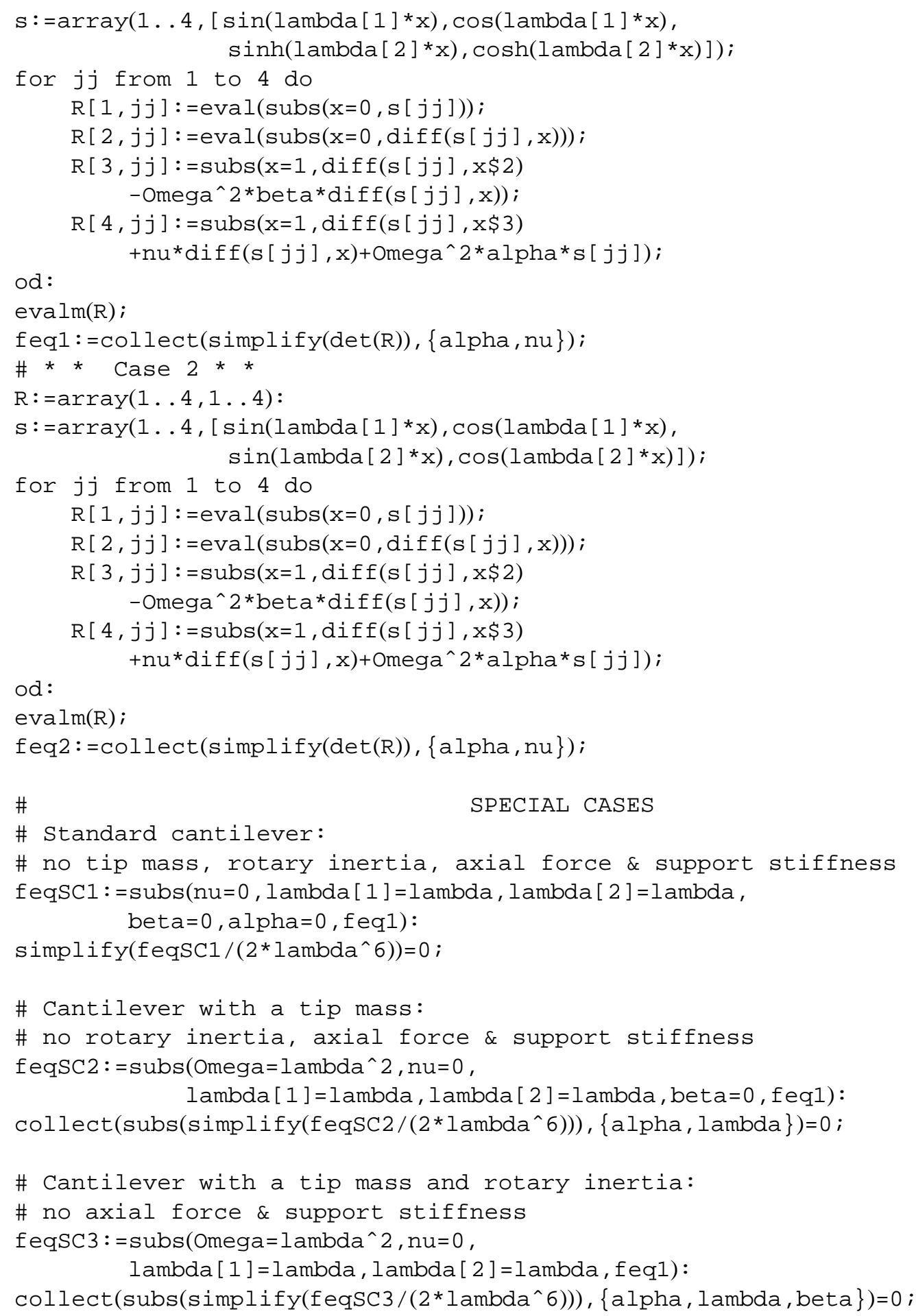

\section{Nomenclature}

$\alpha \quad$ mass ratio, $\alpha=\frac{M}{m L}$

$\beta$ nondimensional rotary inertia, $\beta=\frac{J}{m L^{3}}$ 


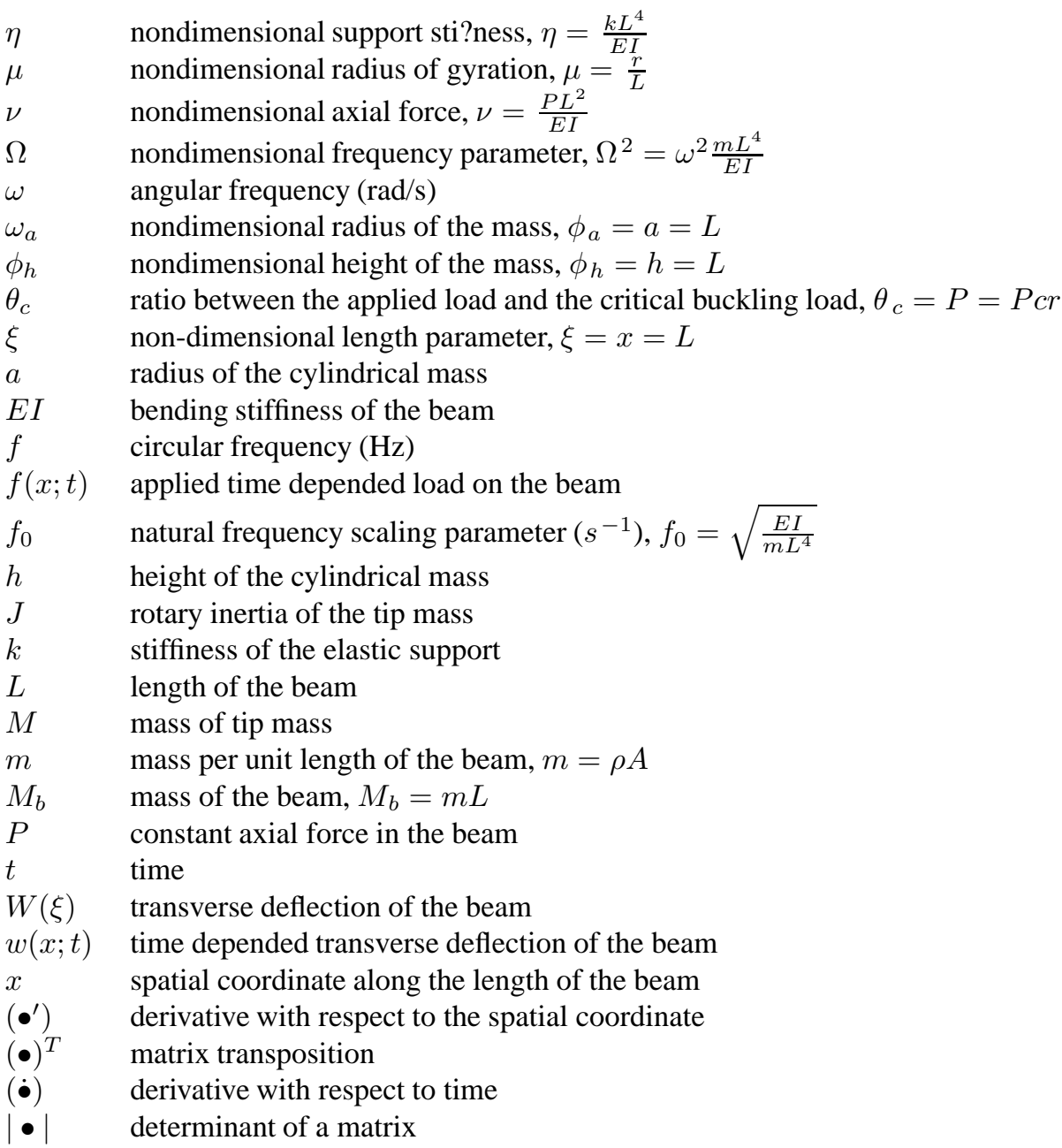

\section{References}

[1] H. Abramovich and O. Hamburger, Vibration of a cantilever timoshenko beam with a tip mass, Journal of Sound and Vibration 148 (1991), $162-170$.

[2] S. Bhattacharya, Pile instability during earthquake liquefaction, Ph.D. thesis, University of Cambridge, Cambridge (U.K.), 2003.

[3] S. Bhattacharya and S. Adhikari, Vibrational characteristics of a piled structure in liquefied soil during earthquakes: Experimental Investigation (Part I) and Analytical Modelling (Part II), Technical Report OUEL 2294/07, Oxford University Engineering Department, Department of Engineering Science, University of Oxford, Oxford, UK, 2007.

[4] S. Bhattacharya and M.D. Bolton, A fundamental omission in seismic pile design leading to collapse, Proceedings of the 11th International Conference on soil dynamics and Earthquake Engineering, Berkeley 1 (2004), 820-827.

[5] S. Bhattacharya, M.D. Bolton and S.P.G. Madabhushi, A reconsideration of the safety of piled bridge foundations in liquefiable soils, Soils and Foundations 45 (2005), 13-25.

[6] S. Bhattacharya, S.P.G. Madabhushi and M.D. Bolton, An alternative mechanism of pile failure in liquefiable deposits during earthquakes, Géotechnique 54 (2004), 203-213.

[7] S. Bhattacharya, S.P.G. Madabhushi and M.D. Bolton, Authors reply to: An alternative mechanism of pile failure in liquefiable deposits during earthquakes, Géotechnique 55 (2005), 259-263.

[8] R.D. Blevins, Formulas for Natural Frequency and Mode Shape, Krieger Publishing Company, Malabar, FL, USA, 1984.

[9] Y. Chen, On the vibration of beams or rods carrying a concentrated mass, Transactions of ASME, Journal of Applied Mechanics 30 (1963), 310-311.

[10] I. Elishakoff, Eigenvalues of Inhomogeneous Structures: Unusual Closed-Form Solutions, CRC Press, Boca Raton, FL, USA, 2005.

[11] I. Elishakoff, Essay on the contributors to the elastic stability theory, Meccanica 40 (2005), 75-110.

[12] I. Elishakoff and V. Johnson, Apparently the first closed-form solution of vibrating inhomogeneous beam with a tip mass, Journal of Sound and Vibration 286 (2005), 1057-1066. 
[13] I. Elishakoff and A. Perez, Design of a polynomially inhomogeneous bar with a tip mass for specified mode shape and natural frequency, Journal of Sound and Vibration 287 (2005), 1004-1012.

[14] M. Géradin and D. Rixen, Mechanical Vibrations, John Wiely \& Sons, New York, NY, second edition, 1997, translation of: Théorie des Vibrations.

[15] D.A. Grant, Effect of rotary inertia and shear deformation on frequency and normal mode equations of uniform beams carrying a concentrated mass, Journal of Sound and Vibration 57 (1978), 357-365.

[16] M. Gurgoze, On the eigenfrequencies of a cantilever beam with attached tip mass and a spring-mass system, Journal of Sound and Vibration 190 (1996), 149-162.

[17] M. Gurgoze, On the eigenfrequencies of a cantilever beam carrying a tip spring-mass system with mass of the helical spring considered, Journal of Sound and Vibration 282 (2005), 1221-1230.

[18] M. Gurgoze and H. Erol, On the frequency response function of a damped cantilever simply supported in-span and carrying a tip mass, Journal of Sound and Vibration 255 (2002), 489-500.

[19] M. Gurgoze and V. Mermertas, On the eigenvalues of a viscously damped cantilever carrying a tip mass, Journal of Sound and Vibration 216 (1998), 309-314.

[20] M. Hetenyi, Beams on Elastic Foundation: Theory with Applications in the Fields of Civil and Mechanical Engineering, University of Michigan Press, Ann Arbor, MI USA, 1946.

[21] S.V. Hoa, Vibration of a rotating beam with tip mass, Journal of Sound and Vibration 67 (1979), 369-391.

[22] T.C. Huang, The effect of rotatory inertia and of shear deformation on the frequency and normal mode equations of uniform beams with simple end conditions, Transactions of ASME, Journal of Applied Mechanics 28 (1961), 579-584.

[23] J.A. Knappett and S.P.G. Madabhushi, Modelling of Liquefaction-induced instability in pile groups, in: Proceedings of 3rd Japan-US workshop on Earthquake Resistant design of lifeline facilities and countermeasures for soil liquefaction, ASCE Special Publication 145.

[24] E. Kreyszig, Advanced engineering mathematics, John Wiley \& Sons, New York, nine edition, 2006.

[25] P.A.A. Laura and R.H. Gutierrez, Vibrations of an elastically restrained cantilever beam of varying cross-section with tip mass of finite length, Journal of Sound and Vibration 108 (1986), 123-131.

[26] R.S. Meera, K. Shanker and P.K. Basudhar, Flexural response of piles under liquefied soil conditions, Geotechnical and Geological Engineering 25 (2007), 409-422.

[27] L. Meirovitch, Principles and Techniques of Vibrations, Prentice-Hall International, Inc., New Jersey, 1997.

[28] M.B. Monagan, K.O. Geddes, K.M. Heal, G. Labahn and S.M. Vorkoetter, Maple 10 Advanced Programming Guide, Maplesoft, Waterloo, Ontario, Canada, 2005

[29] H.R. Oz, Natural frequencies of an immersed beam carrying a tip mass with rotatory inertia, Journal of Sound and Vibration 266 (2003), 1099-1108.

[30] H.H. Pan, Transverse vibration of an Euler-Bernoulli beam carrying a system of heavy bodies, Transactions of ASME, Journal of Applied Mechanics 32 (1965), 434-437.

[31] AIJ, Recommendations for design of building foundations, Architectural Institute of Japan, 2000, in Japanese.

[32] BTL Committee, Study on liquefaction and lateral spreading in the 1995 Hyogoken-Nambu earthquake, Building Research Report No 138, Building Research Institute, Ministry of Construction, Japan, 2000, in Japanese.

[33] A.N. Schofield, Cambridge Centrifuge operations, Géotechnique 30 (1980), 26-268.

[34] A.N. Schofield, Dynamic and Earthquake Geotechnical Centrifuge Modelling, volume 3 of Proceedings of the International Conference Recent Advances in Geotechnical Earthquake Engineering and Soil Dynamics, 1981.

[35] K. Shanker, P.K. Basudhar and N.R. Patra, Buckling of piles under liquefied soil conditions, Geotechnical and Geological Engineering 25 (2007), 303-313.

[36] C.W.S. To, Vibration of a cantilever beam with a base excitation and tip mass, Journal of Sound and Vibration 83 (1982), $445-460$.

[37] K. Tokimatsu and Y. Asaka, Effects of liquefaction-induced ground displacements on pile performance in the 1995 Hyogeken-Nambu earthquake, Special Issue of Soils and Foundations (1998), 163-177.

[38] J.S. Wu and H.M. Chou, Free vibration analysis of a cantilever beam carrying any number of elastically mounted point masses with the analytical-and-numerical-combined method, Journal of Sound and Vibration 213 (1998), 317-332.

[39] J.S. Wu and H.M. Chou, A new approach for determining the natural frequencies and mode shapes of a uniform beam carrying any number of sprung masses, Journal of Sound and Vibration 220 (1999), 451-468.

[40] J.S. Wu and S.H. Hsu, A unified approach for the free vibration analysis of an elastically supported immersed uniform beam carrying an eccentric tip mass with rotary inertia, Journal of Sound and Vibration 291 (2006), 1122-1147.

[41] N. Yoshida and M. Hamada, Damage to foundation piles and deformation pattern of ground due to liquefaction-induced permanent ground deformation, in: Proceedings of 3rd Japan-US workshop on Earthquake Resistant design of lifeline facilities and countermeasures for soil liquefaction, 1996. 

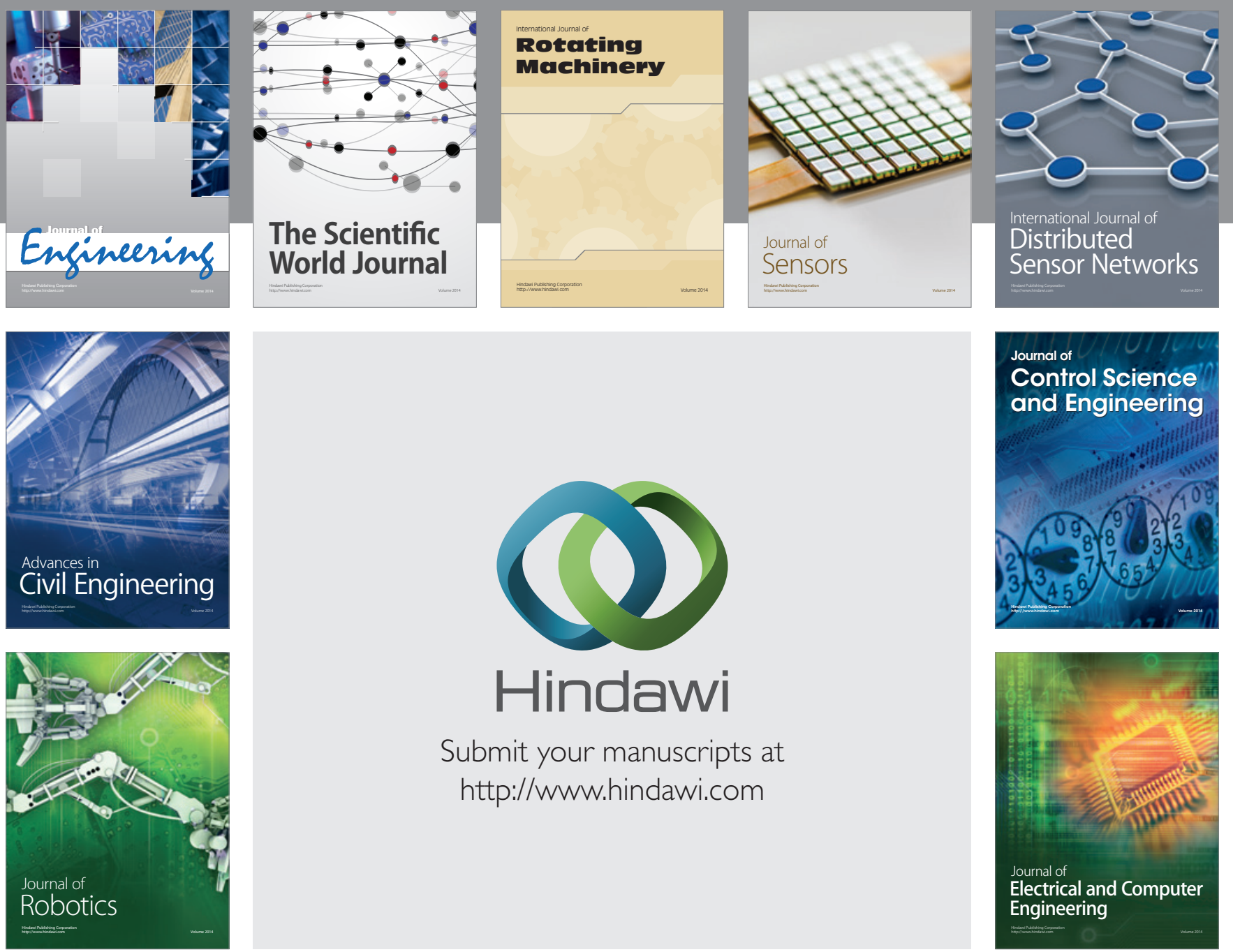

Submit your manuscripts at

http://www.hindawi.com
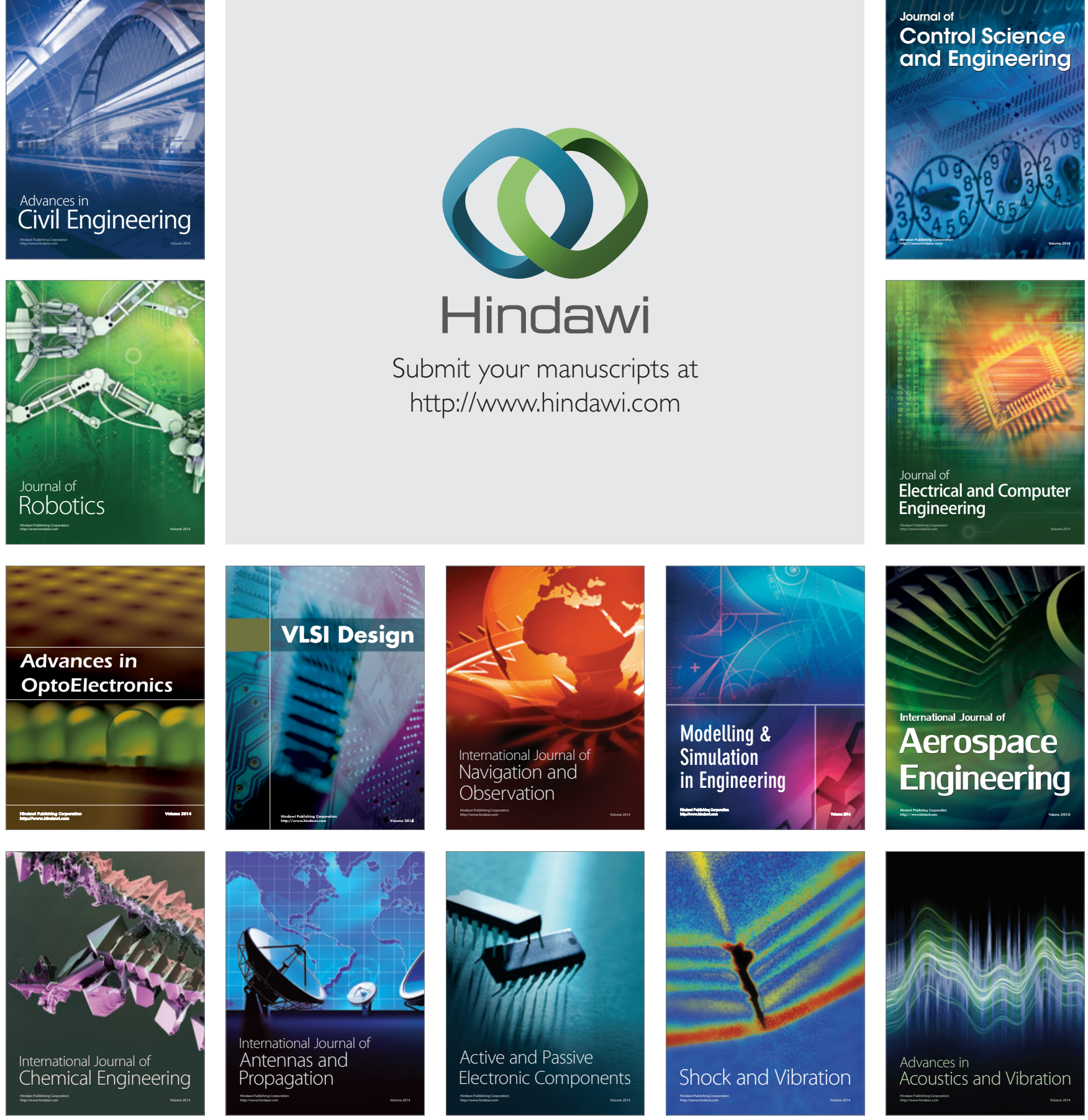